\title{
On approximate reasoning with graded rules *
}

\author{
Martina Daňková \\ Institute for Research and Applications of Fuzzy Modeling, University of Ostrava \\ 30. dubna 22, 70103 Ostrava 1, Czech Republic
}

\begin{abstract}
This contribution presents a comprehensive view on problems of approximate reasoning with imprecise knowledge in the form of a collection of fuzzy IF-THEN rules formalized by approximating formulas of a special type. Two alternatives that follow from the dual character of approximating formulas are developed in parallel. The link to the theory of fuzzy control systems is also explained.
\end{abstract}

Key words: Approximate reasoning, Fuzzy approximation, Normal forms, Fuzzy control systems.

\section{Introduction}

This paper presents a systematic investigation of knowledge-based systems of two special types. The type of a system is determined by interpretation of the available knowledge which can take one of the following two forms:

$$
\begin{array}{ll}
\text { DisK : } & \left(A_{1} \text { and } B_{1}\right) \text { OR } \ldots \text { OR }\left(A_{k} \text { and } B_{k}\right), \\
\text { ConK }: & \left(A_{1} \text { or } B_{1}\right) \text { AND } \ldots \text { AND }\left(A_{k} \text { or } B_{k}\right),
\end{array}
$$

or even simpler

$$
\underbrace{C_{1} \text { OR } \ldots \text { OR } C_{k}}_{\text {DisK }} \text { and } \underbrace{C_{1} \text { AND } \ldots \text { AND } C_{k}}_{\text {ConK }}
$$

where AND (OR) relates to conjunction (disjunction), and (or) relates to strong conjunction (strong disjunction), and $A_{i}, B_{i}, C_{i}, i=1, \ldots, k$, are linguistic

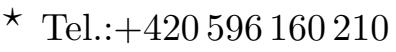

E-mail address: martina.dankova@osu.cz. 
expressions. For the study of these two kinds of formalization, it is suitable to choose a logic, where the strong disjunction is dual to strong conjunction and is definable by means of negation, as is the case of logics with the law of double negation such as Łukasiewicz logic, S-logic [7], IMTL or ŁП [9]. Assuming, e.g. Łukasiewicz logic with the strong conjunction \& and negation $\neg$, where the strong disjunction $\nabla$ is introduced as $\varphi \nabla \psi \equiv_{d f} \neg((\neg \varphi) \&(\neg \psi))$, we obtain $(\varphi \nabla \psi) \leftrightarrow(\neg \varphi \rightarrow \psi)$. Hence, we can rewrite ConK into

\section{ConK2 : (IF [NOT $A_{1}$ ] THEN $\left.B_{1}\right)$ AND $\ldots$ AND (IF [NOT $A_{k}$ ] THEN $B_{k}$ ).}

In this logic, ConK and ConK2 are equivalent and, in addition, they are dual to DisK, which is not generally the case in other logics (e.g. monoidal t-norm based logics see [15] for the overview). We will return to this question in Section 2.2.

Since the main purpose of this work is to follow the results of Perfilieva in [22] (extended in [10]), it is desirable to work in the same logical framework that is BL-logic [17]. Unfortunately in this setting, we are not generally able to introduce the strong disjunction keeping the duality w.r.t. the strong conjunction, because the law of double negation does not hold there. Therefore, it is reasonable to consider only DisK and ConK2 in the sequel, where NOT in ConK2 does not correspond directly to the negation $\neg$ and fulfills here rather symbolical role for representing a negative knowledge.

From the practical point of view, the distinction between various kinds of formalization follows naturally from the requirement to form a description of the given fact as simple as possible. Indeed, some facts are much easier to be described by eliminating inadmissible cases. For example, imagine some decision-making situation, where the operator has to press either $\left\langle\right.$ Button $\left._{1}\right\rangle$ OR ... OR $\left\langle\right.$ Button $\left._{k}\right\rangle$ out of the $k+2$ buttons, whereas the knowledge about not pressing $\left\langle\right.$ Button $\left._{k+1}\right\rangle$ AND $\left\langle\right.$ Button $\left._{k+2}\right\rangle$ yields the same result.

The graded approach (as stated in [21]) seems to be a general principle of the human mind, and therefore we assume the rules DisK and ConK2 to express the fact in consideration using vague predicates. Additionally, there may exist a certain amount of dubiousness over the knowledge base. This is reflected by introducing certain degrees that equip the respective formalization. Such a graded knowledge formalization can be visualized in the spirit of [21] (using the same notation as for evaluated formulae)

$$
\begin{array}{lll}
\text { GradedDisK : } & \langle\text { at most }\rangle f_{1} /\left(A_{1} \text { and } B_{1}\right) \text { OR } \ldots \text { OR }\langle\text { at most }\rangle f_{k} /\left(A_{k} \text { and } B_{k}\right), \\
\text { GradedConK : } & \langle\text { at least }\rangle f_{1} /\left(A_{1} \text { or } B_{1}\right) \text { AND ... AND }\langle\text { at least }\rangle f_{k} /\left(A_{k} \text { or } B_{k}\right),
\end{array}
$$

where $\langle$ at most $\rangle f / A$ means " $A$ is valid at most to the degree $f$ ", analogously, 〈at least $f / A$ reads as " $A$ is valid at least to the degree $f$ ", and the degrees 
$f_{1}, \ldots, f_{k}$ belong to some structure for the truth values. In the sequel, GradedDisK and GradedConK will be called graded rules.

Relating to this topic, the work introduced in [12] and [11] (a brief overview can be found in [14]) may be of the particular interest. Their authors distinguish between gradual rules, certainty rules and their mixture. The gradualness is connected with the properties of implication, certainty with the strong disjunction, and the rules are not equipped with additional truth value as it is in our case. The rules specified by the authors of $[12,11]$ are special cases of our graded rules if we assume the background logic with the law of double negation. There, we simply take all values $f_{i}=0$ (where 0 is associated to the truth constant for the falsity in a structure of truth values) and modify $A_{i}^{\prime}, B_{i}^{\prime}$ by means of involutive negation in CDF.

In this work, we interpret GradedDisK and GradedConK by formulae of predicate fuzzy logic. The weight $f$ is obtained from the latter as well. For example, let us consider a rule

$$
\mathfrak{R} \equiv(x \text { is } \mathcal{A}) \text { and }(y \text { is } \mathcal{B}),
$$

which will be interpreted by a formula $A(x) \& B(y)$. Moreover, we can suppose its verity to be supported by examples $D=\left\{\left(c_{i}, d_{i}\right) \mid i \in I\right\}$, where $\left(c_{i}, d_{i}\right)$ are some object constants of the corresponding language. To each data from $D$ is assigned a weight that impacts the value $f$ in which $\mathfrak{R}$ is satisfied. We may assume that the weight $\omega_{i}$ of $\left(c_{i}, d_{i}\right)$ is given as a truth value of $A\left(c_{i}\right) \& B\left(d_{i}\right)$. Note that $\omega_{i}$ need not be produced necessarily on the basis of definable formula. The final value $f$ of $\mathfrak{R}$ is produced on the basis of knowledge about the data set $D$ with respect to the structure of $\mathfrak{R}$. In this example, we take $f=\max _{i \in I} \omega_{i}$ and we obtain the graded rule $\langle$ at most $\rangle f / \mathfrak{R}$.

Finally, approximate inferences that mimic approximate reasoning in the sense of [26] and cope with graded rules and additional premise $A^{*}$ will be introduced. Inference with graded GradedDisK is based on Zadeh's compositional rule of inference that allows us to derive a consequence of what is known (deduction), i.e. $A^{*}$ and GradedDisK. On the other hand, GradedConK can be used to explain what is known (abduction), i.e. $A^{*}$ and GradedConK. To illustrate the latter, let us consider the following formulae:

$$
\begin{aligned}
A \& B \& A^{*} & \rightarrow B^{*}, \\
A^{*} \& B^{* *} \& A & \rightarrow B .
\end{aligned}
$$

It is known that if (1) is 1-true then the inference schema

$$
(D E): \frac{A^{*}, A \& B}{B^{*}}
$$

is sound (see e.g. [15]) and hence also deductive, i.e. from $\alpha$-true premises we 
derive only $\beta$-true conclusion, where $\alpha \leq \beta$.

Formula (2) relates to the inference rule

$$
(A B): \frac{A, A^{*} \& B^{* *}}{B}
$$

and we wish to find $B^{* *}$ so that $(A B)$ will be sound as well. In other words, we derive $B^{* *}$ as an explanation of $B$ on the basis of preconditions $A$ and $A^{*} \& B^{* *}$, i.e. abductive reasoning. Obviously,

$$
B^{*} \equiv\left[A^{*} \&(A \& B)\right] \quad \text { and } \quad B^{* *} \equiv\left[A^{*} \rightarrow(A \rightarrow B)\right]
$$

fulfil these requirements in any monoidal t-norm based logic. The dual character of GradedDisK and GradedConK together with these two kinds of reasoning lead to the inference rules specified in Section 2.3. The properties of these inferences formulated in Section 3.2 are strongly influenced by those of Hájek in [17], where only the case of GradedDisK is investigated. In this contribution, we extend the results from [10] by adapting Hájek's results to the case of graded GradedDisK and we build the dual approach for graded GradedConK in parallel. Moreover, the whole methodology is in the spirit of [3], which brings the generalization to the results of [10] that create part of Section 3.1. The way of generalization is explained in Remark 2 .

\section{Preliminaries}

\subsection{Many-sorted fuzzy predicate $\operatorname{logic}(B L \forall)$}

The language $J$ with sorts $s_{1}, \ldots, s_{n}$ of $\mathrm{BL} \forall$ includes a non-empty set of predicates of any type, a set of object constants, object variables $x_{i}, y_{i}, z_{i}, \ldots$ of the sorts $s_{i}, i=1, \ldots, n$, a set of connectives $\{\&, \rightarrow\}$, truth constants $\overline{0}$ and $\overline{1} \equiv_{d f} \overline{0} \rightarrow \overline{0}$, quantifiers $\forall, \exists$ and does not include functional symbols. Terms are object variables and object constants.

By a formula, we mean a formula of $\mathrm{BL} \forall$ in the language $J$, build in the usual way, i.e. each $P\left(t_{1}, \ldots, t_{n}\right)$, where $P$ is an $n$-ary predicate and $t_{1}, \ldots, t_{n}$ are terms of the sorts $t_{1}, \ldots, t_{n}$, respectively, and each truth constant is an atomic formula. Each formula results from atomic formulae by iterated use of the following rule: if $\varphi, \psi$ are formulae and $x$ is an object variable then $(\forall x) \varphi$, $(\exists x) \varphi, \varphi \& \psi$ and $\varphi \rightarrow \psi$ are formulae. 
In $\mathrm{BL} \forall$ it is possible to define the following derived connectives:

$$
\begin{array}{crr}
\neg \varphi \quad \text { as } \varphi \rightarrow \overline{0} & \text { negation } \\
\varphi \wedge \psi \text { as } \varphi \&(\varphi \rightarrow \psi) & \text { conjunction } \\
\varphi \vee \psi \text { as }[(\varphi \rightarrow \psi) \rightarrow \psi] \wedge[(\psi \rightarrow \varphi) \rightarrow \varphi] \text { disjunction } \\
\varphi \leftrightarrow \psi \text { as }(\varphi \rightarrow \psi) \&(\psi \rightarrow \varphi) & \text { equivalence }
\end{array}
$$

Before we summarize logical calculus of $\mathrm{BL} \forall$, let us briefly recall that an object constant is always substitutable into a formula and a variable $y$ is substitutable into $\varphi$ for $x$ if the substitution does not turn any free occurrence of $x$ in $\varphi$ into a bound occurrence of $y$.

$\mathrm{BL} \forall$ consists of the following axioms of $\mathrm{BL}$ for connectives:

$$
\begin{aligned}
& (\varphi \rightarrow \psi) \rightarrow[(\psi \rightarrow \chi) \rightarrow(\varphi \rightarrow \chi)], \\
& (\varphi \& \psi) \rightarrow \varphi \\
& {[\varphi \&(\varphi \rightarrow \psi)] \rightarrow[\psi \&(\psi \rightarrow \varphi)],} \\
& {[\varphi \rightarrow(\psi \rightarrow \chi)] \leftrightarrow[(\varphi \& \psi) \rightarrow \chi],} \\
& {[(\varphi \rightarrow \psi) \rightarrow \chi] \rightarrow[((\psi \rightarrow \varphi) \rightarrow \chi) \rightarrow \varphi],} \\
& \overline{0} \rightarrow \varphi
\end{aligned}
$$

together with the axioms on quantifiers:

$$
\begin{aligned}
& (\forall x) \varphi(x) \rightarrow \varphi(t) \quad(t \text { is substitutable for } x \text { in } \varphi), \\
& \varphi(t) \rightarrow(\exists x) \varphi(x) \quad(t \text { is substitutable for } x \text { in } \varphi), \\
& (\forall x)(\varphi \rightarrow \psi) \rightarrow(\varphi \rightarrow(\forall x) \psi) \quad(x \text { is not free in } \varphi), \\
& (\forall x)(\varphi \rightarrow \psi) \rightarrow((\exists x) \varphi \rightarrow \psi) \quad(x \text { is not free in } \psi), \\
& (\forall x)(\varphi \vee \psi) \rightarrow((\forall x) \varphi \vee \psi) \quad(x \text { is not free in } \psi),
\end{aligned}
$$

and two deduction rules

$$
\begin{array}{cl}
\frac{\varphi, \varphi \rightarrow \psi}{\psi} & (\text { modus ponens - MP) } \\
\frac{\varphi}{(\forall x) \varphi} & \text { (generalization - GR }) .
\end{array}
$$

The notions of theory $T$, theory over BL $\forall$, proof and provability in BL $\forall$, proof and provability in a theory over BL $\forall$ are defined in the same way as in classical logic.

Interpretation of the connectives $\{\&, \rightarrow, \vee, \wedge\}$ is given by the corresponding operations $\left\{*, \rightarrow_{*}, \cup, \cap\right\}$, and the constant $\overline{0}$ is interpreted as 0 , which together form a BL-algebra

$$
\mathcal{L}=\left\langle L, \cap, \cup, *, \rightarrow_{*}, 0,1\right\rangle
$$


An $\mathcal{L}$-structure for the language $J$ is of the form

$$
\mathcal{M}=\left\langle\left(D_{i}\right)_{\text {for } s_{i}},\left(r_{P}\right)_{P-\text { predicate }},\left(m_{c}\right)_{c-\text { constant }}\right\rangle,
$$

where $D_{1}, \ldots, D_{n}$ are non-empty sets of objects, $r_{P}$ is an $L$-fuzzy relation of the respective type and $m_{c}$ belongs to $D_{i}$ provided that $c$ is of the type $s_{i}$.

Finally, we summarize useful theorems of BL $\forall$.

Lemma 1 The following are theorems of $B L \forall$ :

$$
\begin{aligned}
& {[\varphi \&(\varphi \rightarrow \psi)] \rightarrow \psi,} \\
& \varphi \rightarrow(\varphi \vee \psi), \\
& (\varphi \rightarrow \psi) \rightarrow[(\varphi \& \chi) \rightarrow(\psi \& \chi)], \\
& (\forall x)(\varphi \rightarrow \psi) \rightarrow((\forall x) \varphi \rightarrow(\forall x) \psi), \\
& (\forall x)(\varphi \rightarrow \psi) \leftrightarrow(\varphi \rightarrow(\forall x) \psi) \quad(x \text { is not free in } \varphi), \\
& (\forall x)(\varphi \rightarrow \psi) \rightarrow((\exists x) \varphi \rightarrow(\exists x) \psi), \\
& (\forall x)(\varphi \rightarrow \psi) \leftrightarrow((\exists x) \varphi \rightarrow \psi) \quad(x \text { is not free in } \psi), \\
& {[(\forall x) \varphi \&(\exists x) \psi] \rightarrow(\exists x)(\varphi \& \psi),} \\
& (\exists x)(\varphi \& \psi) \leftrightarrow((\exists x) \varphi \& \psi) \quad(x \text { is not free in } \psi) .
\end{aligned}
$$

The following remark and conventions relate to the style of presentation of the results throughout the whole paper and should help the reader with an orientation.

Remark 2 How to read the formulae? In the following, we will prove so called graded theorems (for the first time introduced in [16] as stated in [3]). It means that instead of the usual formulation

$$
\text { If } \vdash \varphi \text { then } \vdash \psi \text {, }
$$

we are going to find $n \in \mathbb{N}$ for which

$$
\vdash \varphi^{n} \rightarrow \psi
$$

Whenever we do know $\vdash \varphi^{n} \rightarrow \psi$ and $\vdash \varphi$ then we can derive $\vdash \psi$ easily, however, it is not such a simple case when we proceed the other way round. Hence, the formula $\vdash \varphi^{n} \rightarrow \psi$ is more general and it can be read as

"Formula $\psi$ is valid at least to the degree of $\varphi^{n} . "$

Of course the previous free reading relates to the notion of truth degrees of $\varphi$ and $\psi$ in the particular model.

Notice that the existence of $n$ follows from the deduction theorem (Theorem 2.2.18 in [17]) and the general method of its estimation is related to the concrete proof (or proofs) - the way of using $\varphi$ as an assumption. 


\section{Conventions 1}

(1) How to read the proofs? For the sake of brevity, we will write the proofs as the sequences of formulae $\varphi_{1} \longmapsto \ldots \longmapsto \varphi_{k}$, where $\longmapsto \in\{\longrightarrow, \longleftrightarrow\}$, and for each $i=1, \ldots, k-1$ either $\vdash \varphi_{i} \leftrightarrow \varphi_{i+1}$ if $\longmapsto i s \longleftrightarrow$ or $\vdash \varphi_{i} \rightarrow \varphi_{i+1}$, otherwise.

(2) Provability and truthfulness. The whole work is done on the syntactical level of $B L \forall$ and we will refer to semantical models only in the case of particular examples. Therefore, it is safe to omit the symbol of provability $\vdash$ in $B L \forall$ as well as the symbol for realization $\mathcal{M} \models$ in a model $\mathcal{M}$ in the sequel.

\subsection{Formalization of graded rules}

First, let us recall special formulae (known as normal forms introduced by Perfilieva in [22]), which we will use to formalize collection of fuzzy IF-THEN rules.

Conventions 2 For the sake of brevity, let us denote

$$
\begin{array}{ccc}
{\left[x_{1}, \ldots, x_{n}\right]} & \text { by } & \bar{x} \\
\left\{R_{1}, \ldots, R_{n}\right\} & \text { by } & \bar{R} \\
\left(R_{1}\left(x_{1}, y_{1}\right) \& \cdots \& R_{n}\left(x_{n}, y_{n}\right)\right) & \text { by } & R(\bar{x}, \bar{y})
\end{array}
$$

Analogous shortenings we use for any other binary predicate or variable.

Definition 3 Let $k \in \mathbb{N}$ and $I_{k}=\{1, \ldots, k\}$ and let the language $J_{k}$ be an extension of $J(B L \forall)$ by

(a) a finite set of $n$-tuples of object constants $\left\{\bar{c}_{i}=\left[c_{i_{1}}, \ldots, c_{i_{n}}\right] \mid i \in I_{k}\right\}$, where each $c_{i_{j}}$ is of the sort $s_{j}$ for all $j=1, \ldots, n$,

(b) binary predicate symbols $R_{1}, \ldots, R_{n}$, each $R_{i}$ of the type $\left\langle s_{i}, s_{i}\right\rangle$,

(c) and an $n$-ary predicate $F$ of the type $\left\langle s_{1}, \ldots, s_{n}\right\rangle$.

We define the following formulae

$$
\begin{aligned}
\operatorname{DNF}_{F, k}(\bar{x}) & \equiv_{d f} \bigvee_{i \in I_{k}}\left(R\left(\bar{c}_{i}, \bar{x}\right) \& F\left(\bar{c}_{i}\right)\right), \\
\mathrm{CNF}_{F, k}(\bar{x}) & \equiv_{d f} \bigwedge_{i \in I_{k}}\left(R\left(\bar{x}, \bar{c}_{i}\right) \rightarrow F\left(\bar{c}_{i}\right)\right), \\
\mathrm{DNF}_{F}^{\exists}(\bar{x}) & \equiv_{d f}(\exists \bar{y})(R(\bar{y}, \bar{x}) \& F(\bar{y})), \\
\mathrm{CNF}_{F}^{\forall}(\bar{x}) & \equiv_{d f}(\forall \bar{y})(R(\bar{x}, \bar{y}) \rightarrow F(\bar{y})) .
\end{aligned}
$$


Let $\bar{x}$ consist of all free variables of $\varphi$ and $\psi$, and moreover, $\bar{y}$ be substitutable for $\bar{x}$ in $\varphi$ and $\psi$. We define the following formulae:

$$
\begin{array}{rlll}
\operatorname{Ext}_{\bar{R}} \varphi & \equiv_{d f} & (\forall \bar{x}, \bar{y})[(R(\bar{x}, \bar{y}) \& \varphi(\bar{x})) \rightarrow \varphi(\bar{y})] & \bar{R} \text {-extensionality } \\
\varphi \subseteq \psi & \equiv_{d f} & (\forall \bar{x})(\varphi(\bar{x}) \rightarrow \psi(\bar{x})) & \text { inclusion } \\
\varphi \approx \psi & \equiv_{d f} & (\forall \bar{x})(\varphi(\bar{x}) \leftrightarrow \psi(\bar{x})) & \text { bi-inclusion } \\
\operatorname{Refl}_{R} & \equiv_{d f} & (\forall x) R x x & \text { reflexivity } \\
\operatorname{Sym}_{R} & \equiv_{d f} & (\forall x, y)(R x y \rightarrow R y x) & \text { symmetry } \\
\operatorname{Trans}_{R} & \equiv_{d f} & (\forall x, y, z)[(R x y \& R y z) \rightarrow R x z] & \text { transitivity }
\end{array}
$$

\section{Conventions 3}

(1) Priority of connectives and special symbols. In order to make the formulae more compact, we will reduce the number of brackets inside of formulae by setting the preferences of connectives and special symbols used in Definition 3: 1. The highest priority has $\neg$, afterwards follow 2. $\{\subseteq, \approx\}, 3 .\{\&, \wedge, \vee\}$, and the least priority have $4 .\{\rightarrow, \leftrightarrow\}$.

As an example let us assume the following formula $\varphi \wedge \psi \subseteq \phi \rightarrow \neg \chi \approx \phi$, which stands for $(\varphi \wedge(\psi \subseteq \phi)) \rightarrow((\neg \chi) \approx \phi)$.

(2) Since in some cases, it would become difficult to find over which variables the quantification in $\varphi \subseteq \psi(\varphi \approx \psi)$ is taken, we will use the following notation

$$
\varphi(\bar{x}) \subseteq \psi(\bar{y}) \equiv_{d f}(\forall \bar{x} \bar{y})(\varphi(\bar{x}) \rightarrow \psi(\bar{y}))
$$

and analogously for $\approx$.

(3) For the sake of brevity, we will write only $\mathrm{D}(\mathrm{C}) \mathrm{NF}$ and I instead of $\mathrm{D}(\mathrm{C}) \mathrm{NF}_{F, k}$ and $I_{k}$, respectively, whenever $F$ and $k$ will be clear from the context.

Moreover, let Prop $=\{$ Refl, Sym, Trans $\}$ then we will write

$$
\operatorname{Prop}_{\bar{R}} \quad \text { for } \quad \operatorname{Prop}_{R_{1}} \& \ldots \& \operatorname{Prop}_{R_{n}} \text {. }
$$

\section{Remark 4}

(1) The formulae $\mathrm{DNF}_{F, k}$ and $\mathrm{CNF}_{F, k}$ have their roots in algebraic expressions of the classical disjunctive and conjunctive normal forms for Boolean function (see $§$ 5.2 in [21]). Let us recall that each Boolean function (domain and range 
is $\{0,1\}) f$ of $n$ variables can be represented in the following forms

$$
\begin{aligned}
f_{\mathrm{DNF}}(\bar{x}) & =0 \vee \bigvee_{\bar{y} \in\{0,1\}^{n}} x_{1}^{y_{1}} \wedge \ldots \wedge x_{n}^{y_{n}} \wedge f(\bar{y}), \\
f_{\mathrm{CNF}}(\bar{x}) & =1 \wedge \bigwedge_{\bar{y} \in\{0,1\}^{n}} x_{1}^{\urcorner^{y_{1}}} \vee \ldots \vee x_{n}^{\neg y_{n}} \vee f(\bar{y}) \\
& =1 \wedge \bigwedge_{\bar{y} \in\{0,1\}^{n}} x_{1}^{y_{1}} \wedge \ldots \wedge x_{n}^{y_{n}} \rightarrow f(\bar{y}), \text { so that } \\
f_{\mathrm{DNF}}(\bar{x}) & =f_{\mathrm{CNF}}(\bar{x})=f(\bar{x}),
\end{aligned}
$$

where $x^{y}=\left\{\begin{array}{l}\neg x, y=0 ; \\ x, \text { otherwise, }\end{array}\right.$ and $\mathfrak{B}=\langle\{0,1\}, \wedge, \vee, \neg, 0,1\rangle$ is Boolean algebra. We can rewrite these expressions by means of equivalence by simple observation: $x^{y}=x \leftrightarrow y$. This observation is important for the future generalization: let us assume that $\mathcal{L}$ is of the form (14) and we deal with fuzzy relations (in the particular realization) instead of Boolean functions, moreover, the parts including elementary equivalences are replaced with binary fuzzy relations using which we describe neighborhoods of the respective nodes $y_{i}$ and these elementary parts are joined by generalized operations $\left(*, \rightarrow_{*}\right)$ to create an $n$-dimensional information about $\bar{y}$. Finally, we use the lattice operations $(\vee, \wedge)$ to create the global expression. If we transform $f_{\mathrm{D}(\mathrm{C}) \mathrm{NF}}$ directly then the received expression relates to logical formula $\mathrm{DNF}_{F}^{\exists}\left(\mathrm{CNF}_{F}^{\forall}\right)$. And if we intentionally choose the set of nodes $\left\{\bar{y}_{i}\right\}_{i \in I}$, hence we obtain expressions relating to $\mathrm{DNF}_{F, k}$ and $\mathrm{CNF}_{F, k}$.

(2) Notice that $\mathrm{DNF}_{F}^{\exists}$ and $\mathrm{CNF}_{F}^{\forall}$ are known in literature as image and preimage of $F$ in relation $R$, see e.g. [24]. Here, these formulae can be viewed as some kind of "limit" case of $\mathrm{DNF}_{F, k}$ and $\mathrm{CNF}_{F, k}$, respectively, when we proceed over the all elements of the universe relating to the respective realization.

Let us assume $n=2$. Then a partial knowledge formalized by (24) can be interpreted as a rule-base consisting of $k$ graded rules in the following form

$$
\left\{\langle\text { at most }\rangle f_{i} /\left(x \text { is } \mathcal{A}_{i}\right) \text { and }\left(y \text { is } \mathcal{B}_{i}\right)\right\}_{i \in I}
$$

where $\left(x\right.$ is $\left.\mathcal{A}_{i}\right)$ is formalized by $R_{1}\left(c_{i_{1}}, x_{1}\right),\left(y\right.$ is $\left.\mathcal{B}_{i}\right)$ by $R_{2}\left(c_{i_{2}}, x_{2}\right)$, and the degree $f_{i}$ by $F\left(c_{i_{1}}, c_{i_{2}}\right)$. Moreover, the part "at most to the degree" is realized using \& in DNF. Indeed, since \& is in residuated lattice interpreted by $*$, the latter immediately follows from the inequality $x * y \leq y$ for all $x, y$ from the respective support. Hence, the truth value of the $i$ 'th disjunct in DNF will never exceed the truth value associated to $F\left(c_{i_{1}}, c_{i_{2}}\right)$ for an arbitrary $\mathcal{M}$ valuation of the object variables. 
Analogously, the formula (25) formalizes

$$
\left\{\langle\text { at least }\rangle f_{i} /\left(x \text { is } \mathcal{C}_{i}\right) \text { and }\left(y \text { is } \mathcal{D}_{i}\right)\right\}_{i \in I},
$$

where $\left(x\right.$ is $\left.\mathcal{C}_{i}\right)$ is now formalized by $R_{1}\left(x_{1}, c_{i_{1}}\right),\left(y\right.$ is $\left.\mathcal{D}_{i}\right)$ by $R_{2}\left(x_{2}, c_{i_{2}}\right)$, and the degree $f_{i}$ by $F\left(c_{i_{1}}, c_{i_{2}}\right)$. The part "at least to the degree" is connected with $\rightarrow$ in CNF. Observe that in any residuated lattice $\rightarrow$ is interpreted by $\rightarrow_{*}$ and the inequality $y \leq x \rightarrow_{*} y$ holds for all $x, y$. Therefore, the truth value of the $i$ 'th conjunct in CNF will never fall under the truth value associated to $F\left(c_{i_{1}}, c_{i_{2}}\right)$.

Above, we have spoken about the gradualness only w.r.t. fuzzy rules and it needs to be pointed out that on the level of syntax, we deal with (24) and (25), i.e. the formulae in the usual sense.

Let us consider a very simple problem to illustrate the way of using normal forms to formalize gradual rules.

Example 4 Let $\mathcal{L}$ be standard product algebra $\left\langle[0,1], \cap, \cup, \odot, \rightarrow_{\odot}, 0,1\right\rangle$ and $\mathcal{M}=\langle M=[0.1,0.9], f, r, 0.25,0.75\rangle$ be an $\mathcal{L}$-structure for the language $J_{2}$, where $f, r, 0.25,0.75$ interpret $F, R_{1}, c_{1}, c_{2}$, respectively. Let $f$ be a fuzzy relation on $M$ depicted on Figure 1 by the dashed line. Furthermore,

$$
r(x, y)=(x \rightarrow \odot y)^{k(x, y)} \odot(y \rightarrow \odot x)^{l(x, y)}
$$

where $k, l: M^{2} \rightarrow \mathbb{N}$. Then the realization of the disjunctive normal form in $\mathcal{M}$ gives us the following expression

$$
\begin{aligned}
{\left[\left(0.25 \rightarrow_{\odot} x\right)^{0} \odot(x \rightarrow \odot 0.25)^{4} \odot f(0.25)\right] \cup } & \\
& {\left[\left(0.75 \rightarrow_{\odot} x\right)^{6} \odot(x \rightarrow \odot 0.75)^{1} \odot f(0.75)\right]=} \\
& \left(x \rightarrow_{\odot} 0.25\right)^{4} \cup\left[\left(0.75 \rightarrow_{\odot} x\right)^{6} \odot\left(x \rightarrow_{\odot} 0.75\right)\right]
\end{aligned}
$$

which can be seen on Figure 1(a) as the solid line. If we assume that $\preceq_{i}$ are symbols representing fuzzy orderings $(x \rightarrow \odot y)^{i}, i \in \mathbb{N}$, then the above expression corresponds with the graded rules

$$
\text { 〈at most } \left.\rangle 1 /\left(x \preceq_{4} 0.25\right) \quad \text { OR } \quad \text { lat most }\right\rangle 1 /\left(0.75 \preceq_{6} x\right) \text { and }\left(x \preceq_{1} 0.75\right) .
$$

Now, let us consider $\mathcal{N}$ be an $\mathcal{L}$-structure for $J_{1}$, which is identical with $\mathcal{M}$ up to the interpretation of the object constant $c_{1}$, which we choose as 0.43 . Hence the interpretation of $\mathrm{CNF}_{F, 1}$ in $\mathcal{N}$ has the form

$$
\left[(x \rightarrow \odot 0.43)^{9} \odot\left(0.43 \rightarrow_{\odot} x\right)^{11}\right] \rightarrow_{\odot} 0.013
$$

see Figure 1(b). And analogously as in the case of $\mathrm{DNF}$, we can rewrite it into 


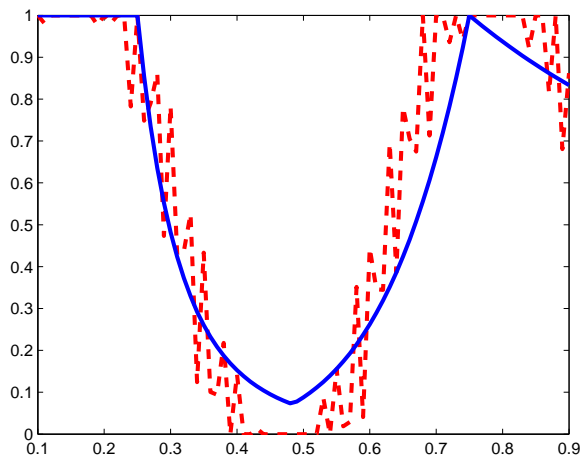

(a) Model of $\mathrm{DNF}_{F}$ (solid line) for $F$ (dashed line)

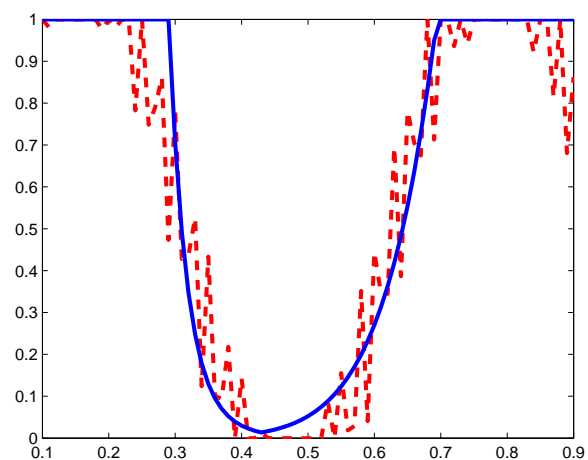

(b) Model of $\mathrm{CNF}_{F}$ (solid line) for $F$ (dashed line)

Fig. 1. Model of approximate description from Example 4.

the gradual rule

$$
\text { 〈at least }>0.013 /\left(x \preceq{ }_{9} 0.43\right) \text { and }\left(0.43 \preceq_{11} x\right) \text {. }
$$

Observe that even in the case of one rule CNF does not turn to DNF and vice-versa.

In various applications of fuzzy logic, the following two basic types of rules are used

$$
\begin{array}{r}
\left\{\left(x \text { is } \mathcal{A}_{i}\right) \text { and }\left(y \text { is } \mathcal{B}_{i}\right)\right\}_{i \in I}, \quad(M A M D) \\
\left\{\operatorname{IF}\left(x \text { is } \mathcal{C}_{i}\right) \text { THEN }\left(y \text { is } \mathcal{D}_{i}\right)\right\}_{i \in I},
\end{array}
$$

where $\mathcal{A}_{i}, \mathcal{B}_{i}, \mathcal{C}_{i}, \mathcal{D}_{i}$ are usually interpreted as fuzzy sets forming a boundary for the variables $x, y$, respectively.

Let us now explain, how these two kinds of rules are related to their graded variants. Note that each rule-base of the form (MAMD) turns to (32) simply by taking all $f_{i}=1$ and it is formalized by (24). In the case of (IMPL), the situation is more complicated. Let us assume a background logic with the law of double negation. Then (IMPL) of the form

$$
\left\{\operatorname{IF}\left(x \text { is } \operatorname{NOT} \mathcal{A}_{i}\right) \operatorname{THEN}\left(y \text { is } \mathcal{B}_{i}\right)\right\}_{i \in I},
$$

is equivalent to

$$
\left\{\left(x \text { is } \mathcal{A}_{i}\right) \text { or }\left(y \text { is } \mathcal{B}_{i}\right)\right\}_{i \in I},
$$

which naturally turns into $(33)$ by taking all $f_{i}=0$, i.e.

$$
\left\{\left\langle\text { at least }>0 /\left(x \text { is } \operatorname{NOT} \mathcal{A}_{i}\right) \text { and }\left(y \text { is } \operatorname{NOT} \mathcal{B}_{i}\right)\right\}_{i \in I} .\right.
$$

The properties of NOT interpreted as $\neg$ are connected with the relationship between (24) and (25) expressed by means of $\neg$. Inside of the above specified 
background logic, we can show that

$$
\begin{aligned}
\neg \mathrm{DNF}_{\neg F, k}(\bar{x}) \longleftrightarrow \bigwedge_{i \in I_{k}} \neg\left(R\left(\bar{c}_{i}, \bar{x}\right) \& \neg F\left(\bar{c}_{i}\right)\right) & \longleftrightarrow \\
\bigwedge_{i \in I_{k}}\left(R\left(\bar{c}_{i}, \bar{x}\right)\right. & \left.\rightarrow \neg \neg F\left(\bar{c}_{i}\right)\right) \longleftrightarrow \mathrm{CNF}_{F, k}(\bar{x}) .
\end{aligned}
$$

There is an interesting relationship between these formulae even without involving negation, as will be shown later in Subsection 3.1.

Note that the concept of CNF as a formalization of the eliminative knowledge outlined at the beginning of this paper works only in the case of logic with the law of double negation for $\neg$. In general, $\neg$ has not such a good properties and, moreover, we are not able to define disjunction dual to \& (corresponding to t-conorm on the semantical level). This is the reason why we use CNF in its implicative form. The following proposition shows under which conditions (left sides of implications) we can prove $((\varphi \rightarrow \psi) \rightarrow \psi) \leftrightarrow \varphi$. Observe that this formula is closely related to the law of double negation, i.e. $((\varphi \rightarrow \overline{0}) \rightarrow$ $\overline{0}) \leftrightarrow \varphi$.

Proposition 5 Let $\vdash_{G}, \vdash_{L}, \vdash_{P}$ denote provability in Gödel, Eukasiewicz, Product logic, respectively. Then

$$
\begin{aligned}
\vdash \neg \psi & \rightarrow[(\varphi \rightarrow \psi) \leftrightarrow \neg \varphi] \\
\vdash \varphi & \rightarrow((\varphi \rightarrow \psi) \rightarrow \psi) \\
\vdash_{G} \varphi \wedge \psi & \rightarrow[((\varphi \rightarrow \psi) \rightarrow \psi) \leftrightarrow \varphi] \\
\vdash_{L}(\psi \rightarrow \varphi) & \rightarrow[((\varphi \rightarrow \psi) \rightarrow \psi) \leftrightarrow \varphi] \\
\vdash_{P} \neg \neg \psi \&(\varphi \leftrightarrow \psi) & \rightarrow[((\varphi \rightarrow \psi) \rightarrow \psi) \leftrightarrow \varphi]
\end{aligned}
$$

PROOF: (36) Obviously $\neg \varphi \rightarrow(\varphi \rightarrow \psi)$ and moreover by (3), we obtain $\neg \psi \rightarrow[(\varphi \rightarrow \psi) \rightarrow \neg \varphi]$, which together proves the claim.

(37) By exchange in $(\varphi \rightarrow \psi) \rightarrow(\varphi \rightarrow \psi)$.

(38) The following is provable in Gödel logic

$$
\varphi \wedge(\varphi \leftrightarrow \psi) \longleftrightarrow(\varphi \wedge \psi) \wedge(\varphi \rightarrow \psi) \longleftrightarrow \varphi \wedge \psi
$$

Since the instance of (17) is

$$
(\psi \rightarrow \varphi) \&[(\varphi \rightarrow \psi) \rightarrow \psi] \rightarrow[(\psi \&(\varphi \rightarrow \psi)) \rightarrow(\varphi \& \psi)]
$$

and $\varphi \& \psi \rightarrow \varphi$, we have $\psi \&(\varphi \leftrightarrow \psi) \rightarrow[((\varphi \rightarrow \psi) \rightarrow \psi) \rightarrow \varphi]$, which together with (37) and (41) proves (38). 
(39) In Eukasiewicz logic $[(\varphi \rightarrow \psi) \rightarrow \psi] \rightarrow[(\psi \rightarrow \varphi) \rightarrow \varphi]$ is provable. The proof of (39) follows by exchange in the above formula and adding (37).

(40) In Product logic, we have cancelation law, i.e.

$$
\neg \neg \psi \rightarrow[(\varphi \& \psi \rightarrow \phi \& \psi) \rightarrow(\varphi \rightarrow \phi)],
$$

hence also

$$
\begin{aligned}
(\psi \rightarrow \varphi) \&[(\varphi \rightarrow \psi) \rightarrow \psi] \longrightarrow & \\
\psi & \&(\varphi \rightarrow \psi) \rightarrow \varphi \& \psi \longrightarrow \neg \neg \psi \rightarrow((\varphi \rightarrow \psi) \rightarrow \varphi),
\end{aligned}
$$

from which we get $\neg \neg \psi \&(\psi \leftrightarrow \varphi) \&[(\varphi \rightarrow \psi) \rightarrow \psi] \rightarrow \varphi$.

Example 5 On the semantical level, the situation looks as follows:

(1) In the case of Gödel implication $x \rightarrow_{G} y=\left\{\begin{array}{l}1, x \leq y ; \\ y, \text { otherwise. }\end{array}\right.$ we have

$$
\left(x \rightarrow_{G} y\right) \rightarrow_{G} y=\left\{\begin{array}{l}
y, x \leq y \\
1, \text { otherwise }
\end{array}\right.
$$

Relating to the formula (38), we see that it is not possible to restore the original value $x$ up to the case when it is equal to $y$. Hence, it is suitable only for the crisp sets. This is very bad for our idea of replacing the double negation $(\varphi \rightarrow \overline{0}) \rightarrow \overline{0}$ by double $\bar{p}$-negation $(\varphi \rightarrow \bar{p}) \rightarrow \bar{p}$ for the dealing with fuzzy sets.

(2) For the Eukasiewicz implication $x \rightarrow_{L} y=1 \wedge(1-x+y)$, we have $\left(x \rightarrow_{L} y\right) \rightarrow_{L} y=x \vee y$.

(3) For the Product implication $x \rightarrow_{P} y=\left\{\begin{array}{l}1, x \leq y ; \\ \frac{y}{x}, \text { otherwise. }\end{array}\right.$

we have

$$
\left(x \rightarrow_{P} y\right) \rightarrow_{P} y=\left\{\begin{array}{l}
y, x \leq y \\
1, \frac{y}{x} \leq b \\
x, \text { otherwise }
\end{array}\right.
$$

To keep the dual concept of the descriptions, we propose to interpret a particular rule in CNF, e.g. $\varphi \rightarrow \bar{p}$ as "nearly not $\varphi$ " on the basis of (36) (take $\bar{p}$ as $\psi$ ), where the "nearness" is related to $\neg \bar{p}$. This reading may be used for all systems of knowledge that is to be formalized by CNF. 


\subsection{Inference with graded rules}

Approximate inference rule is often considered as a basis for dealing with fuzzy IF-THEN rules and a non-precise input knowledge. A generalized rule of modus ponens as a particular case of compositional rule of inference in the global concept of many-valued logics has been introduced by L. Zadeh in [27]. The analysis of logical aspects of Zadeh's compositional rules of inference was done by P. Hájek in [17] or V. Novák in [19-21] (for evaluated syntax). From the other works let us mention e.g. [23,25,13]. Inference rules were also intensively studied from the algebraical point of view as special operations called compositions (see, e.g., $[18,14,4,6]$ ).

In the sequel, we will assume $J_{F C}=J_{k} \cup\left\{A^{*}\right\}$, where $A^{*}$ is a predicate of the type $\left\langle s_{1}, \ldots, s_{p}\right\rangle$.

Let us suppose $n>1, p \in \mathbb{N}$ such that $1 \leq p \leq n-1$, and $\bar{x}_{p}=\left[x_{1}, \ldots, x_{p}\right]$, $\bar{y}_{p}=\left[x_{p+1}, \ldots, x_{n}\right]$. Then, we define

$$
\begin{aligned}
& B_{\mathrm{DNF}}^{*}\left(\bar{y}_{p}\right) \equiv_{d f}\left(\exists \bar{x}_{p}\right)\left(A^{*}\left(\bar{x}_{p}\right) \& \mathrm{DNF}_{F, k}(\bar{x})\right), \\
& B_{\mathrm{CNF}}^{*}\left(\bar{y}_{p}\right) \equiv_{d f}\left(\forall \bar{x}_{p}\right)\left(A^{*}\left(\bar{x}_{p}\right) \rightarrow \mathrm{CNF}_{F, k}(\bar{x})\right),
\end{aligned}
$$

which define $B_{\mathrm{DNF}}^{*}$ and $B_{\mathrm{CNF}}^{*}$ from $A^{*}$ using the Zadeh's compositional rule of inference and Bandler-Kouhout's product (BK-product [2]), respectively. Since BK-product can be viewed as a dual to Zadeh's composition, and moreover, $\mathrm{CNF}_{F, k}$ is dual to $\mathrm{DNF}_{F, k}$, we conclude that also $B_{\mathrm{CNF}}^{*}$ is in a certain sense dual to $B_{\mathrm{DNF}}^{*}$. Let us demonstrate the duality on the special example of CNF and DNF in the case of Eukasiewicz logic, where the following sequence of equivalences is provable:

$$
\begin{aligned}
B_{\mathrm{CNF}}^{*}(y) \longleftrightarrow & (\forall x)\left[A^{*}(x) \rightarrow \bigwedge_{i \in I}\left(R_{1}\left(x, c_{i}\right) \rightarrow \neg R_{2}\left(y, d_{i}\right)\right)\right] \longleftrightarrow \\
& (\forall x)\left[A^{*}(x) \rightarrow \neg \bigvee_{i \in I}\left(R_{1}\left(x, c_{i}\right) \& R_{2}\left(y, d_{i}\right)\right)\right] \longleftrightarrow \\
& \neg(\exists x)\left[A^{*}(x) \& \bigvee_{i \in I}\left(R_{1}\left(x, c_{i}\right) \& R_{2}\left(y, d_{i}\right)\right)\right] \longleftrightarrow \neg B_{\mathrm{DNF}}^{*}(y),
\end{aligned}
$$

where $x, y$ are variables of the respective sorts and each $c_{i}, d_{i}, i \in I$ are object constants of the respective sorts.

Approximate inferences based on (42) and (43) can be visualized as inference rules of the following forms

$$
(D E) \quad \frac{A^{*}, \mathrm{DNF}_{F, k}}{B_{\mathrm{DNF}}^{*}} \text { and }(A B) \frac{A^{*}, B_{\mathrm{CNF}}^{*}}{\mathrm{CNF}_{F, k}}
$$

Here, $F$ represents an ideal situation that can be expressed as $\mathrm{DNF}_{F}$ and $\mathrm{CNF}_{F}$. Later, we will see that for the extensional $F$, we can prove $F \rightarrow \mathrm{CNF}_{F}$ 
and $\mathrm{DNF}_{F} \rightarrow F$. At this point, we can formulate the following problem: Find $B^{*}$ such that

$(a)$

$$
\begin{aligned}
& A^{*} \& B^{*} \rightarrow F, \\
& A^{*} \& B^{*} \rightarrow \mathrm{CNF}_{F},
\end{aligned}
$$$$
\text { and there exists no } B^{\prime} \text { : }
$$

$$
\left(B^{*} \rightarrow B^{\prime}\right) \&\left(A^{*} \& B^{\prime} \rightarrow \mathrm{CNF}_{F}\right)
$$

$$
F \rightarrow\left(A^{*} \rightarrow B^{*}\right)
$$$$
\mathrm{DNF}_{F} \rightarrow\left(A^{*} \rightarrow B^{*}\right)
$$

and there exists no $B^{\prime}$ :

$\left(\mathrm{DNF}_{F} \rightarrow\left(A^{*} \rightarrow B^{\prime}\right)\right) \&\left(B^{\prime} \rightarrow B^{*}\right)$,

$B^{\prime}$ is different from $B^{*}$. In fact, the formulae $B_{\mathrm{CNF}}^{*}$ and $B_{\mathrm{DNF}}^{*}$ are solutions to the problem (a) and (b), respectively. Since we deal with the extensional $F$ then the first formulae in (a) and (b) follow from (71) and (70). The second ones are obvious by adjunction property (6), and the last requirements are fulfilled by observing

$$
\begin{aligned}
\left(B_{\mathrm{CNF}}^{*} \rightarrow B^{\prime}\right) \&\left(A^{*} \& B^{\prime} \rightarrow \mathrm{CNF}_{F}\right) & \rightarrow\left(B_{\mathrm{CNF}}^{*} \leftrightarrow B^{\prime}\right), \\
\left(\mathrm{DNF}_{F} \rightarrow\left(A^{*} \rightarrow B^{\prime}\right)\right) \&\left(B^{\prime} \rightarrow B_{\mathrm{DNF}}^{*}\right) & \rightarrow\left(B_{\mathrm{DNF}}^{*} \leftrightarrow B^{\prime}\right) .
\end{aligned}
$$

Notice that multiple application of (DE) (to $\left.A^{*}\left(\bar{x}_{p}\right) \leftrightarrow R\left(\bar{c}_{i_{p}}, \bar{x}_{p}\right)\right)$ and (AB) (to $A_{i}^{*}\left(\bar{x}_{p}\right) \equiv_{d f} R\left(\bar{x}_{p}, \bar{c}_{i_{p}}\right)$ ) for $i \in I$ allow us to create new formulae

$$
\begin{aligned}
& \bigvee_{i \in I}\left(A_{i}^{*}\left(\bar{x}_{p}\right) \& B_{\mathrm{CNF}, i}^{*}\left(\bar{y}_{p}\right)\right), \\
& \bigwedge_{i \in I}\left(A_{i}^{*}\left(\bar{x}_{p}\right) \rightarrow B_{\mathrm{DNF}, i}^{*}\left(\bar{y}_{p}\right)\right),
\end{aligned}
$$

where $B_{\mathrm{D}(\mathrm{C}) \mathrm{NF}, i}^{*}\left(\bar{y}_{p}\right)$ denotes the respective formulae $B_{\mathrm{D}(\mathrm{C}) \mathrm{NF}}^{*}\left(\bar{y}_{p}\right)$ with $A^{*} \leftrightarrow$ $A_{i}^{*}$. For the future, it would be interesting to investigate the differences between $B_{\mathrm{CNF}, i}^{*}\left(\bar{y}_{p}\right)\left(B_{\mathrm{DNF}, i}^{*}\left(\bar{y}_{p}\right)\right)$ and $R\left(\bar{d}_{i_{p}}, \bar{y}_{p}\right)\left(R\left(\bar{y}_{p}, \bar{d}_{i_{p}}\right)\right)$, where $\bar{d}_{i_{p}}=\left[c_{i_{p+1}}, \ldots, c_{i_{n}}\right]$.

Later, we will investigate properties of fuzzy systems based on graded rules (32) formalized by $\mathrm{D}(\mathrm{C}) \mathrm{NF}$ with an appropriate rule of inference without taking into account fuzzification and defuzzification methods.

\section{Fuzzy systems based logical approximation}

\subsection{Approximating formulae}

In this subsection, we will mostly reformulate the results from [10] in accordance with a methodology manifested in [3]. These results are in the scope of the logical approximation, which is a theory aiming at studying properties of a class of formulae in an simplified form relating to some initial formula. There, 
the significant role is played by a formula of the form $\epsilon \rightarrow\left(\varphi \leftrightarrow \varphi_{S}\right)$ called conditional equivalence. We may interpret it as a lower bounded or graded equivalence between the given formula $\varphi$ and its simplified version $\varphi_{S}$.

As pointed in [22], if we consider a standard model then the conditional equivalence expresses a precision of approximation of " $\varphi$ " by " $\varphi_{S}$ ". To prove a conditional equivalence for normal forms, we must in addition, require the initial formula to be extensional. Recall that this property is natural for each propositional formula and in the case of formulae containing extensional predicates, we have the extensionality for the whole formula as well (see Lemma 5.6.8 in $[17])$.

Without considering the extensionality, we are able to prove only the following relationship between both normal forms.

Lemma 6 Let $m \in \mathbb{N}, m<k$, and $C_{k}$ denote $(\forall \bar{x}) \bigvee_{i \in I_{k}}\left(R\left(\bar{x}, \bar{c}_{i}\right) \& R\left(\bar{c}_{i}, \bar{x}\right)\right)$. Moreover, let us define

$$
\begin{aligned}
& D_{i}(x, y) \equiv \equiv_{d f} \bigwedge_{j \in I}\left(R_{i}\left(c_{j_{i}}, x\right) \rightarrow R_{i}\left(c_{j_{i}}, y\right)\right), \\
& E_{i}(x, y) \equiv_{d f} \bigwedge_{j \in I}\left(R_{i}\left(y, c_{j_{i}}\right) \rightarrow R_{i}\left(x, c_{j_{i}}\right)\right) .
\end{aligned}
$$

Then the following is provable:

$$
\begin{aligned}
\mathrm{DNF}_{F, m} & \subseteq \mathrm{DNF}_{F, m+1}, \\
\mathrm{CNF}_{F, m+1} & \subseteq \mathrm{CNF}_{F, m}, \\
C_{k} \rightarrow \mathrm{CNF}_{F, k} & \subseteq \mathrm{DNF}_{F, k}, \\
\operatorname{Ext}_{\bar{D}} \mathrm{DNF}_{F, k}, & \operatorname{Ext}_{\bar{E}} \mathrm{CNF}_{F, k}, \\
\operatorname{Trans}_{\bar{R}} & \rightarrow \operatorname{Ext}_{\bar{R}} \mathrm{DNF}_{F, k}, \\
\operatorname{Trans}_{\bar{R}} & \rightarrow \operatorname{Ext}_{\bar{R}} \mathrm{CNF}_{F, k} .
\end{aligned}
$$

PROOF: (46) - (47) are obvious.

(48) From (15), it follows that

$$
\begin{aligned}
& R\left(\bar{x}, \bar{c}_{i}\right) \&\left(R\left(\bar{x}, \bar{c}_{i}\right) \rightarrow F\left(\bar{c}_{i}\right)\right) \rightarrow F\left(\bar{c}_{i}\right) \\
& R\left(\bar{x}, \bar{c}_{i}\right) \& R\left(\bar{c}_{i}, \bar{x}\right) \&\left(R\left(\bar{x}, \bar{c}_{i}\right) \rightarrow F\left(\bar{c}_{i}\right)\right) \rightarrow R\left(\bar{c}_{i}, \bar{x}\right) \& F\left(\bar{c}_{i}\right) \\
& R\left(\bar{x}, \bar{c}_{i}\right) \& R\left(\bar{c}_{i}, \bar{x}\right) \& \bigwedge_{i \in I}\left(R\left(\bar{x}, \bar{c}_{i}\right) \rightarrow F\left(\bar{c}_{i}\right)\right) \rightarrow \bigvee_{i \in I}\left(R\left(\bar{c}_{i}, \bar{x}\right) \& F\left(\bar{c}_{i}\right)\right) \\
& \bigvee_{i \in I}\left(R\left(\bar{x}, \bar{c}_{i}\right) \& R\left(\bar{c}_{i}, \bar{x}\right)\right) \& \bigwedge_{i \in I}\left(R\left(\bar{x}, \bar{c}_{i}\right) \rightarrow F\left(\bar{c}_{i}\right)\right) \rightarrow \bigvee_{i \in I}\left(R\left(\bar{c}_{i}, \bar{x}\right) \& F\left(\bar{c}_{i}\right)\right) .
\end{aligned}
$$


(49) By (17) and (6), it follows

$$
\begin{aligned}
R\left(\bar{c}_{i}, \bar{x}\right) \& F\left(\bar{c}_{i}\right) \longrightarrow & \left(\left(R\left(\bar{c}_{i}, \bar{x}\right) \rightarrow R\left(\bar{c}_{i}, \bar{y}\right)\right) \rightarrow R\left(\bar{c}_{i}, \bar{y}\right) \& F\left(\bar{c}_{i}\right)\right) \longrightarrow \\
\bigvee_{i \in I}\left[\left(R\left(\bar{c}_{i}, \bar{x}\right)\right.\right. & \left.\left.\rightarrow R\left(\bar{c}_{i}, \bar{y}\right)\right) \rightarrow R\left(\bar{c}_{i}, \bar{y}\right) \& F\left(\bar{c}_{i}\right)\right] \longrightarrow \\
\bigwedge_{i \in I}\left(R\left(\bar{c}_{i}, \bar{x}\right)\right. & \left.\rightarrow R\left(\bar{c}_{i}, \bar{y}\right)\right) \rightarrow \bigvee_{i \in I}\left(R\left(\bar{c}_{i}, \bar{y}\right) \& F\left(\bar{c}_{i}\right)\right),
\end{aligned}
$$

and

$$
\begin{aligned}
D(\bar{x}, \bar{y}) & \longleftrightarrow D_{1}\left(x_{1}, y_{1}\right) \& \ldots \& D_{n}\left(x_{n}, y_{n}\right) \longleftrightarrow \\
\bigwedge_{i_{1} \ldots i_{n} \in I} & {\left[\left(R_{1}\left(c_{i_{1}}, x_{1}\right) \rightarrow R_{1}\left(c_{i_{1}}, y_{1}\right)\right) \& \ldots \&\left(R_{n}\left(c_{i_{n}}, x_{n}\right) \rightarrow R_{n}\left(c_{i_{n}}, y_{n}\right)\right)\right] \longrightarrow } \\
& \bigwedge_{i_{1} \ldots i_{n} \in I}\left[R_{1}\left(c_{i_{1}}, x_{1}\right) \& \ldots \& R_{n}\left(c_{i_{n}}, x_{n}\right) \rightarrow R_{1}\left(c_{i_{1}}, y_{1}\right) \& \ldots \& R_{n}\left(c_{i_{n}}, y_{n}\right)\right] \longrightarrow \\
& \bigwedge_{i \in I}\left(R\left(\bar{c}_{i}, \bar{x}\right) \rightarrow R\left(\bar{c}_{i}, \bar{y}\right)\right),
\end{aligned}
$$

hence we have proved $\operatorname{Ext}_{\bar{D}} \mathrm{DNF}_{F, k}$.

(50) Analogous.

(51) - (52) Obviously, $P \subseteq R \rightarrow\left(\operatorname{Ext}_{R} S \rightarrow \operatorname{Ext}_{P} S\right)$. The claims follow by $\operatorname{Trans}_{\bar{R}} \rightarrow \operatorname{Trans}_{R_{i}}$ and $R_{i} \subseteq D_{i}$ as well as $R_{i} \subseteq E_{i}$ for all $i \in I$.

From (49) and (50), we see that for the logical approximation of $F$ we will use the extensional formulae. Moreover, the degree of inclusion DNF in CNF is bounded from below by the degree relating to a composition of a domain covering qualities (partition of the input and output space) of both normal forms expressed by $C_{k}$, (see (48)), which we check over the whole universe of the respective model. For the recent advances in formal theory of fuzzy partitions see [8].

Involving the extensionality property allows us to prove the following formulae.

Theorem 7

$$
\begin{aligned}
\operatorname{Ext}_{\bar{R}} F & \rightarrow \mathrm{DNF}_{F, k} \subseteq F, \\
\operatorname{Ext}_{\bar{R}} F & \rightarrow F \subseteq \mathrm{CNF}_{F, k}, \\
\operatorname{Ext}_{\bar{R}} F \& C_{k} & \rightarrow \mathrm{DNF}_{F, k} \approx F, \\
\operatorname{Ext}_{\bar{R}} F \& C_{k} & \rightarrow \mathrm{CNF}_{F, k} \approx F, \\
\operatorname{Refl}_{\bar{R}} & \rightarrow\left(\operatorname{Ext}_{\bar{R}} F \leftrightarrow \mathrm{DNF}_{F}^{\exists} \approx F\right), \\
\operatorname{Refl}_{\bar{R}} & \rightarrow\left(\operatorname{Ext}_{\bar{R}} F \leftrightarrow \mathrm{CNF}_{F}^{\forall} \approx F\right) .
\end{aligned}
$$

\section{PROOF:}


(53)

$$
\begin{aligned}
\operatorname{Ext}_{\bar{R}} F \rightarrow & (\forall \bar{x})\left(R\left(\bar{c}_{i}, \bar{x}\right) \& F\left(\bar{c}_{i}\right) \rightarrow F(\bar{x})\right) \text { for all } i \in I, \text { hence } \\
\operatorname{Ext}_{\bar{R}} F \longrightarrow & \bigwedge_{i \in I}(\forall \bar{x})\left(R\left(\bar{c}_{i}, \bar{x}\right) \& F\left(\bar{c}_{i}\right) \rightarrow F(\bar{x})\right) \longleftrightarrow \\
& (\forall \bar{x}) \bigwedge_{i \in I}\left(R\left(\bar{c}_{i}, \bar{x}\right) \& F\left(\bar{c}_{i}\right) \rightarrow F(\bar{x})\right) \longrightarrow \\
& (\forall \bar{x})\left[\bigvee_{i \in I}\left(R\left(\bar{c}_{i}, \bar{x}\right) \& F\left(\bar{c}_{i}\right)\right) \rightarrow F(\bar{x})\right] \longleftrightarrow \text { DNF } \subseteq F .
\end{aligned}
$$

(54) Analogously

$$
\begin{aligned}
\operatorname{Ext}_{\bar{R}} F \longrightarrow & (\forall \bar{x}) \bigwedge_{i \in I}\left(F(\bar{x}) \rightarrow\left(R\left(\bar{c}_{i}, \bar{x}\right) \rightarrow F\left(\bar{c}_{i}\right)\right)\right) \longrightarrow \\
& (\forall \bar{x})\left(F(\bar{x}) \rightarrow \bigwedge_{i \in I}\left(R\left(\bar{c}_{i}, \bar{x}\right) \rightarrow F\left(\bar{c}_{i}\right)\right)\right) \longleftrightarrow F \subseteq \mathrm{CNF} .
\end{aligned}
$$

(55) We prove the formula using (48) and (54)

$$
\begin{aligned}
\operatorname{Ext}_{\bar{R}} F \& C_{k} \longrightarrow & (F(\bar{x}) \subseteq \operatorname{CNF}(\bar{x})) \&(\operatorname{CNF}(\bar{x}) \subseteq \operatorname{DNF}(\bar{x})) \longrightarrow \\
F(\bar{x}) \subseteq \operatorname{DNF}(\bar{x}) \longrightarrow \text { by }(53) F & \approx \mathrm{DNF} .
\end{aligned}
$$

(56) Analogous to (55).

$(57)$

$$
\begin{aligned}
\operatorname{Ext}_{\bar{R}} F \longleftrightarrow & (\forall \bar{x}, \bar{y})(R(\bar{y}, \bar{x}) \& F(\bar{y}) \rightarrow F(\bar{x})) \longleftrightarrow \\
& (\forall \bar{x})[(\exists \bar{y})(R(\bar{y}, \bar{x}) \& F(\bar{y})) \rightarrow F(\bar{x})] \longleftrightarrow \operatorname{DNF}_{F}^{\exists}(\bar{x}) \subseteq F(\bar{x}), \\
\operatorname{Refl}_{\bar{R}} \longrightarrow & \left(\overline{1} \rightarrow \operatorname{Refl}_{\bar{R}} \longrightarrow(\forall \bar{x})[F(\bar{x}) \rightarrow F(\bar{x}) \& R(\bar{x}, \bar{x})] \longrightarrow\right. \\
& (\forall \bar{x})[F(\bar{x}) \rightarrow(\exists \bar{y})(R(\bar{y}, \bar{x}) \& F(\bar{y}))] \longleftrightarrow F(\bar{x}) \subseteq \operatorname{DNF}_{F}^{\exists}(\bar{x}), \\
F(\bar{x}) \approx & \operatorname{DNF}_{F}^{\exists}(\bar{x}) \longrightarrow \operatorname{DNF}_{F}^{\exists}(\bar{x}) \subseteq F(\bar{x}) \longleftrightarrow \operatorname{Ext}_{\bar{R}} F, \\
\operatorname{Refl}_{\bar{R}} \rightarrow & {\left[\operatorname{Ext}_{\bar{R}} F \leftrightarrow F \approx \mathrm{DNF}_{F}^{\exists}\right] . }
\end{aligned}
$$

(58) Analogous to (57).

Due to the extensionality property, we are able to show that

$$
\begin{aligned}
& \mathrm{DNF}_{F, k} \subseteq F,\left(\text { " } \mathrm{DNF}_{F, k} \text { " creates a lower approximation of " } F "\right), \\
& F \subseteq \mathrm{CNF}_{F, k},\left(\text { " } \mathrm{CNF}_{F, k} \text { " is an upper approximation of " } F "\right) \text {. }
\end{aligned}
$$

And moreover, $F \approx \mathrm{D}(\mathrm{C}) \mathrm{NF}_{F, k}$ is determined by choice of $\bar{c}_{i}$ and $\bar{R}$ in a particular model, as it can be seen from $C_{k}$. 


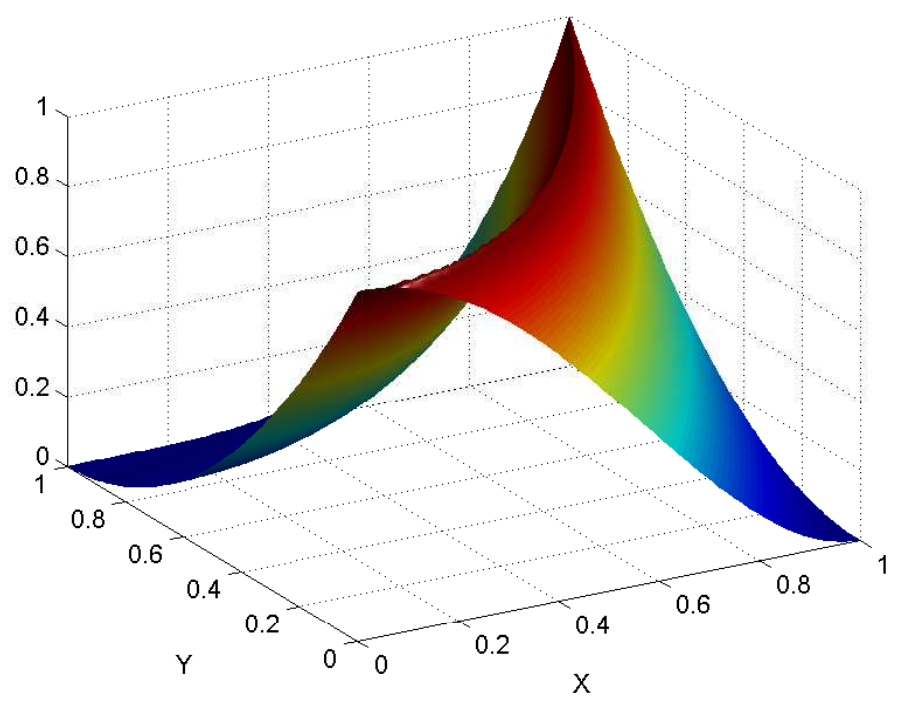

Fig. 2. Relation to be approximated from Example 6.7.

By $(46)+(53)$ and $((47)+(54))$, we see that the sequence of approximating formulae $\mathrm{DNF}_{F}, k$ for increasing $k$ approaches $F$ and in the limit case, it coincide with $F$ whenever $\bar{R}$ is reflexive (57), (58). Note that we can find semantical proofs (not in graded form and under additional conditions required from $\bar{R}$ ) of (57) and (58) in [5] or [4].

It follows from (55) and (56), that we can efficiently approximate only extensional formulae. Below, we show some examples.

Example 6 Let us consider a set $D=\left\{\bar{d}_{i} \mid i \in J\right\}$ of examples. Then the following can be demonstrated:

(1) $F_{\mathrm{DNF}}(\bar{x}) \longleftrightarrow \overline{0} \vee \bigvee_{i \in J} R\left(\bar{x}, \bar{d}_{i}\right)$ is extensional w.r.t. $\bar{Q}$, where each

$$
Q_{i}(x, y) \equiv_{d f} \bigwedge_{j \in J}\left(R\left(x, d_{j_{i}}\right) \rightarrow R\left(y, d_{j_{i}}\right)\right) .
$$

(2) $F_{\mathrm{CNF}}(\bar{x}) \longleftrightarrow \overline{1} \wedge \bigwedge_{i \in J} R\left(\bar{d}_{i}, \bar{x}\right)$ is extensional w.r.t. $\bar{Q}^{\prime}$, where each

$$
Q_{i}^{\prime}(x, y) \equiv_{d f} \bigwedge_{j \in J}\left(R\left(d_{j_{i}}, x\right) \rightarrow R\left(d_{j_{i}}, y\right)\right) .
$$

(3) $\operatorname{Trans}_{\bar{R}} \rightarrow \operatorname{Ext}_{R} F_{\mathrm{DNF}}$ and $\operatorname{Trans}_{\bar{R}} \rightarrow \operatorname{Ext}_{R} F_{\mathrm{CNF}}$.

(4) Let $\kappa$ be a truth constant that extends $J_{k}$ and we assume that

$$
\vdash\left(\forall \bar{x}_{p}\right)\left[\bigvee_{i \in J} R\left(\bar{x}_{p}, \bar{d}_{i_{p}}\right) \leftrightarrow \kappa\right]
$$

then

$$
F_{1}(\bar{x}) \longleftrightarrow \overline{0} \vee\left[\bigvee_{i \in J} R\left(\bar{x}_{p}, \bar{d}_{i_{p}}\right) \rightarrow \bigvee_{i \in J} R\left(\bar{x}, \bar{d}_{i}\right)\right]
$$




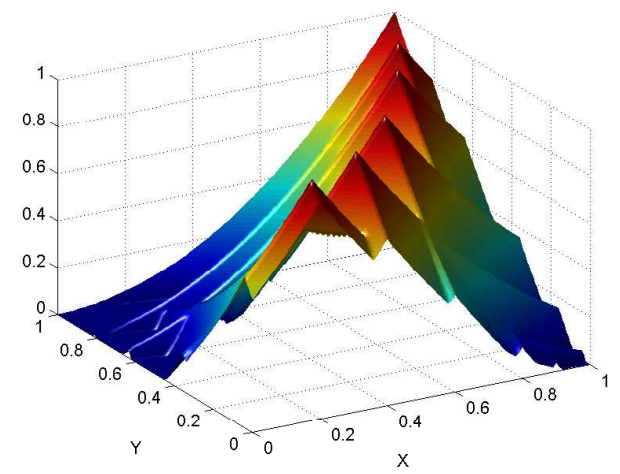

(a) $\operatorname{DNF}_{F, 6}(x, y)$

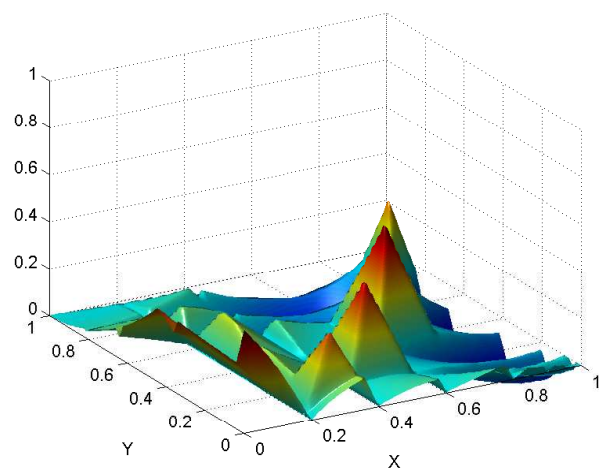

(b) Error of approximation by DNF

Fig. 3. Approximation of the relation from Figure 2 by DNF.

is extensional w.r.t. $\bar{Q}$.

(5) Let $\kappa$ be as above and again $\vdash\left(\forall \bar{x}_{p}\right)\left[\bigvee_{i \in J} R\left(\bar{d}_{i_{p}}, \bar{x}_{p}\right) \leftrightarrow \kappa\right]$ then

$$
F_{2}(\bar{x}) \longleftrightarrow \overline{1} \wedge\left[\bigwedge_{i \in J} R\left(\bar{d}_{i_{p}}, \bar{x}_{p}\right) \rightarrow \bigwedge_{i \in J} R\left(\bar{d}_{i}, \bar{x}\right)\right]
$$

is extensional w.r.t. $\bar{Q}^{\prime}$.

(6) Let $f$ be a functional symbol and $\approx_{1}$ and $\approx_{2}$ be binary predicates for which we will write $x \approx_{1(2)} y$ instead of $\approx_{1(2)}(x, y)$, where $x, y$ are variables of the respective sorts. The following formula expresses a property of $f$ that is called compatibility w.r.t. $\approx_{1}$ and $\approx_{2}$ (see [4])

$$
C o m \approx_{1,2} f \equiv_{d f}(\forall x, y)\left[\left(x \approx_{1} y\right) \rightarrow\left(f(x) \approx_{2} f(y)\right)\right]
$$

where $x, y$ are of the sort $s, f(x), f(y)$ are of the sort $s^{\prime}$ and $\approx_{1}\left(\approx_{2}\right)$ is of the type $\langle s, s\rangle\left(\left\langle s^{\prime}, s^{\prime}\right\rangle\right)$. Define $F$ as follows

$$
F_{f}(x, y) \equiv_{d f} y \approx_{2} f(x) .
$$

Then, it is easy to prove that

$$
\operatorname{Comp}_{\approx_{1,2}} f \& \mathrm{Sym}_{\approx_{2}} \&\left(\operatorname{Trans}_{\approx_{2}}\right)^{2} \rightarrow \mathrm{Ext}_{\approx_{1,2}} F_{f},
$$

and additionally, we are able to show the property of functionality of $F_{f}$ (expressed by (62)) saying that any two images of the same input $x$ are "close enough"

$$
\begin{gathered}
\operatorname{Func}_{(\approx)} F \equiv_{d f}\left(\forall x, y, y^{\prime}\right)\left[F(x, y) \& F\left(x, y^{\prime}\right) \rightarrow y \approx y^{\prime}\right], \\
\operatorname{Trans}_{\approx_{2}} \& \operatorname{Sym}_{\approx_{2}} \rightarrow \operatorname{Func}_{\left(\approx_{2}\right)} F_{f} .
\end{gathered}
$$

(7) For illustration of the above example, we will assume that the logical connectives are interpreted by the corresponding operations of the standard Eukasiewicz algebra $\mathcal{L}=\langle[0,1], \otimes, \Rightarrow, 0,1\rangle$. Moreover, let $\left\{f, F_{f}, \approx_{1}, \approx_{2}\right\}$ 


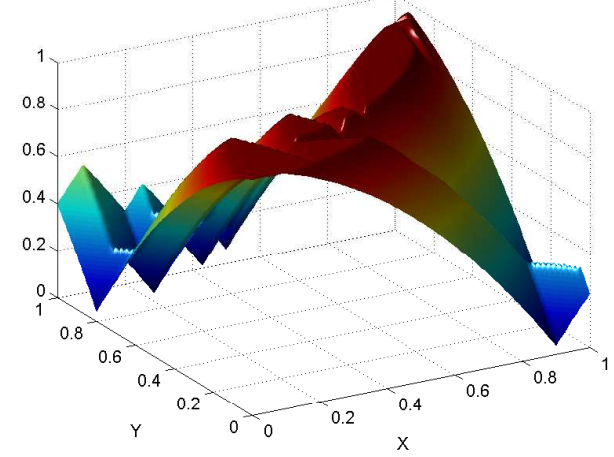

(a) $\mathrm{CNF}_{F, 6}(x, y)$

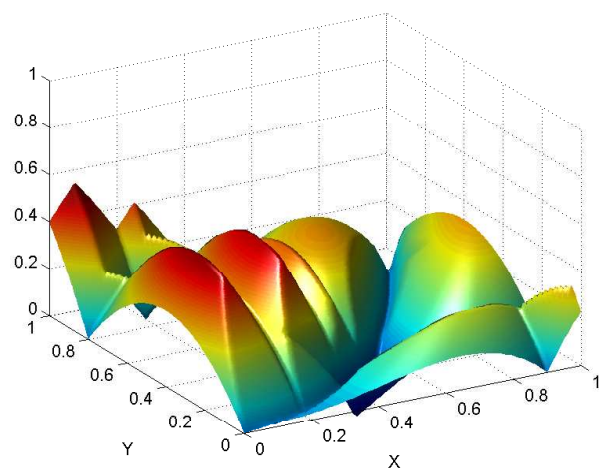

(b) Error of approximation by CNF

Fig. 4. Approximation of the relation from Figure 2 by CNF.

be interpreted as $\left\{\tilde{f}, \tilde{F}_{f}, \cong, \cong\right\}$, respectively, where $\tilde{f}=x^{2}, x \cong y=$ $(1-|x-y|)^{2}$ and $M=[0,1]$.

In this case, $\cong$ is similarity relation (reflexive, symmetric, transitive) and $\tilde{F}_{f}$ (see Figure 2) is a fuzzy function in Hájek's sense ([17]), i.e. $\tilde{F}_{f}$ is extensional w.r.t. $\cong$ (since for all $x, y \in M:\left|x^{2}-y^{2}\right| \leq|x-y|$, hence $x \cong y \leq x^{2} \cong y^{2}$ ) and maps the same inputs to indistinguishable outputs due to (62). Approximation given by $\mathrm{DNF}_{F_{f}}$ is shown on Figure 3 and similarly, approximation by $\mathrm{CNF}_{F_{f}}$ on Figure 4. The difference between these two approximations is evident from Figure 5. There, the nodes suitable for approximating formulae are depicted (circles for DNF and diamonds for $\mathrm{CNF}$ ). It is clear that nodes in which we construct DNF (CNF) lay in local maxima (minima).

In $(\S 7,[17])$, examples of $F$ from $D=\left\{\left(c_{i}, d_{i}\right) \mid i \in I\right\}$ are assumed to be 1-true, i.e., the formula $\bigwedge_{i \in J} F\left(c_{i}, d_{i}\right)$ is taken as an axiom and approximating formulae for fuzzy function $F$ (see Example 6.7) are defined as

$$
\begin{aligned}
& \operatorname{MAMD}(x, y) \equiv \equiv_{d f} \bigvee_{i \in I}\left[\left(x \approx_{1} c_{i}\right) \&\left(y \approx_{2} d_{i}\right)\right], \\
& \operatorname{RULES}(x, y) \equiv_{d f} \bigwedge_{i \in I}\left[\left(x \approx_{1} c_{i}\right) \rightarrow\left(y \approx_{2} d_{i}\right)\right]
\end{aligned}
$$

Notice that MAMD and DNF constructed in the examples from $D$ are identical, unlike RULES and CNF as it can be seen from Figure 6 and Figure 4.

\subsection{Properties of approximate inferences}

The following results are analogous to those given in [17] for Zadeh's compositional rule of inference and IF-THEN rules of the Mamdani type (formalized 


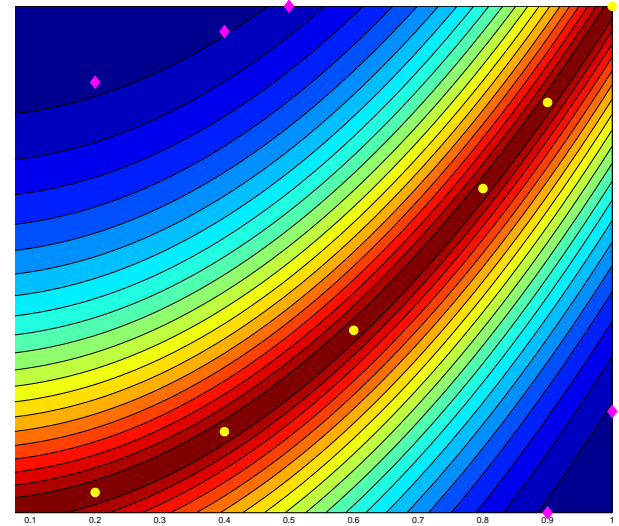

Fig. 5. Nodes marked by circles (diamonds) in which approximating formula $\mathrm{DNF}(\mathrm{CNF})$ is constructed.

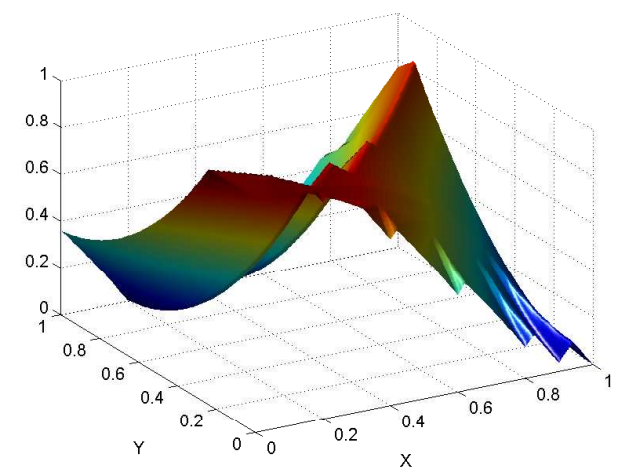

(a) $\operatorname{RULES}(x, y)$

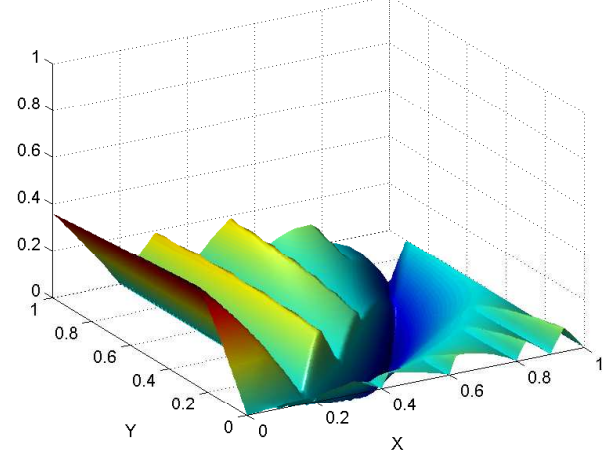

(b) Error of approximation by RULES

Fig. 6. Approximation of the relation from Figure 2 by means of the formula RULES. as MAMD). First, we will focus on the relationship between outputs of approximate inferences.

Proposition 8 Under the notation introduced in Section 2.3

$$
\begin{aligned}
(\forall \bar{x}) \operatorname{DNF}_{F, k}(\bar{x}) & \rightarrow\left[\left(\exists \bar{x}_{p}\right) A^{*}\left(\bar{x}_{p}\right) \rightarrow\left(\forall \bar{y}_{p}\right) B_{\mathrm{DNF}}^{*}\left(\bar{y}_{p}\right)\right], \\
\left(\forall \bar{x}_{p}\right) A^{*}\left(\bar{x}_{p}\right) \&\left(\forall \bar{y}_{p}\right) B_{\mathrm{CNF}}^{*}\left(\bar{y}_{p}\right) & \rightarrow(\forall \bar{x}) \mathrm{CNF}_{F, k}(\bar{x}), \\
\left(\operatorname{Ext}_{\bar{R}} F\right)^{2} \&\left(\forall \bar{x}_{1}, \bar{x}_{2}\right) D\left(\bar{x}_{1}, \bar{x}_{2}\right) & \rightarrow B_{\mathrm{DNF}}^{*}\left(\bar{y}_{1_{p}}\right) \subseteq B_{\mathrm{CNF}}^{*}\left(\bar{y}_{2_{p}}\right), \\
C_{k} \&\left(\exists \bar{x}_{p}\right)\left(A^{*}\left(\bar{x}_{p}\right)\right)^{2} & \rightarrow B_{\mathrm{CNF}}^{*}\left(\bar{y}_{p}\right) \subseteq B_{\mathrm{DNF}}^{*}\left(\bar{y}_{p}\right), \\
\left(\operatorname{Ext}_{\bar{R}} F\right)^{2} & \rightarrow B_{\mathrm{DNF}}^{*}\left(\bar{y}_{p}\right) \subseteq B_{\mathrm{CNF}}^{*}\left(\bar{y}_{p}\right), \\
C_{k} \&\left(\exists \bar{x}_{p}\right)\left(A^{*}\left(\bar{x}_{p}\right)\right)^{2} \&\left(\operatorname{Ext}_{\bar{R}} F\right)^{2} & \rightarrow B_{\mathrm{DNF}}^{*}\left(\bar{y}_{p}\right) \approx B_{\mathrm{CNF}}^{*}\left(\bar{y}_{p}\right),
\end{aligned}
$$

for arbitrary $p \in \mathbb{N}, 1 \leq p<n$.

PROOF: (64) - (65) Obvious. 
(66) By (53), (54) and (49), we have

$$
\begin{aligned}
\left(\operatorname{Ext}_{\bar{R}} f\right)^{2} \&\left(\forall \bar{x}_{1}, \bar{x}_{2}\right) D\left(\bar{x}_{1}, \bar{x}_{2}\right) \longrightarrow\left(\forall \bar{x}_{1}, \bar{x}_{2}\right)\left(\operatorname{DNF}\left(\bar{x}_{1}\right) \rightarrow \operatorname{CNF}\left(\bar{x}_{2}\right)\right) \longrightarrow \\
\quad\left(\forall \bar{x}_{1}, \bar{x}_{2}\right)\left[A^{*}\left(\bar{x}_{1_{p}}\right) \& A^{*}\left(\bar{x}_{2_{p}}\right) \& \operatorname{DNF}\left(\bar{x}_{1}\right) \rightarrow \operatorname{CNF}\left(\bar{x}_{2}\right)\right] \longrightarrow \\
\left(\forall \bar{x}_{1}, \bar{x}_{2}\right)\left[A^{*}\left(\bar{x}_{1_{p}}\right) \& \operatorname{DNF}\left(\bar{x}_{1}\right) \rightarrow\left(A^{*}\left(\bar{x}_{2_{p}}\right) \rightarrow \operatorname{CNF}\left(\bar{x}_{2}\right)\right)\right] \longrightarrow \\
\left(\forall \bar{y}_{1_{p}}, \bar{y}_{2_{p}}\right)\left[\left(\exists \bar{x}_{1_{p}}\right) A^{*}\left(\bar{x}_{1_{p}}\right) \& \operatorname{DNF}\left(\bar{x}_{1}\right) \rightarrow\left(\forall \bar{x}_{2_{p}}\right)\left(A^{*}\left(\bar{x}_{2_{p}}\right) \rightarrow \operatorname{CNF}\left(\bar{x}_{2}\right)\right)\right] .
\end{aligned}
$$

(67) Let $C_{k}(\bar{x})$ denote $\bigvee_{i \in I_{k}}\left(R\left(\bar{x}, \bar{c}_{i}\right) \& R\left(\bar{c}_{i}, \bar{x}\right)\right)$. By (48) and transitivity ax$\operatorname{iom}(3)$

$$
\begin{gathered}
\left(A^{*}\left(\bar{x}_{p}\right) \rightarrow \operatorname{CNF}(\bar{x})\right) \longrightarrow A^{*}\left(\bar{x}_{p}\right) \rightarrow\left(C_{k}(\bar{x}) \rightarrow \operatorname{DNF}(\bar{x})\right) \longrightarrow \\
A^{*}\left(\bar{x}_{p}\right) \rightarrow\left[C_{k}(\bar{x}) \rightarrow \operatorname{DNF}(\bar{x})\right] \&\left[A^{*}\left(\bar{x}_{p}\right) \rightarrow A^{*}\left(\bar{x}_{p}\right)\right] \longrightarrow \\
A^{*}\left(\bar{x}_{p}\right) \rightarrow\left[C_{k}(\bar{x}) \& A^{*}\left(\bar{x}_{p}\right) \rightarrow \operatorname{DNF}(\bar{x}) \& A^{*}\left(\bar{x}_{p}\right)\right] \longleftrightarrow \\
\left(A^{*}\left(\bar{x}_{p}\right)\right)^{2} \& C_{k}(\bar{x}) \rightarrow \operatorname{DNF}(\bar{x}) \& A^{*}\left(\bar{x}_{p}\right), \\
\left(\forall \bar{x}_{p}\right)\left(A^{*}\left(\bar{x}_{p}\right) \rightarrow \operatorname{CNF}(\bar{x})\right) \rightarrow \\
{\left[\left(\exists \bar{x}_{p}\right)\left(A^{*}\left(\bar{x}_{p}\right)\right)^{2} \& C_{k}(\bar{x}) \rightarrow\left(\exists \bar{x}_{p}\right) \operatorname{DNF}(\bar{x}) \& A^{*}\left(\bar{x}_{p}\right)\right],} \\
\left(\forall \bar{y}_{p}\right)\left(\exists \bar{x}_{p}\right)\left(A^{*}\left(\bar{x}_{p}\right)\right)^{2} \& C_{k}(\bar{x}) \rightarrow\left(\forall \bar{y}_{p}\right)\left[B_{\mathrm{CNF}}^{*}\left(\bar{y}_{p}\right) \rightarrow B_{\mathrm{DNF}}^{*}\left(\bar{y}_{p}\right)\right] .
\end{gathered}
$$

(68) From (66) and reflexivity of $D$.

(69) By (67) and (68).

As one would expect from the relationship between $\mathrm{D}(\mathrm{C}) \mathrm{NF}$ and $F$, it is not completely true that $B_{\mathrm{DNF}}^{*} \subseteq B_{\mathrm{CNF}}^{*}$ (formulae $(66),(68)$ ) nor $B_{\mathrm{CNF}}^{*} \subseteq B_{\mathrm{DNF}}^{*}$ (67). From (68), it follows that the extensionality is essential, and the part $\left(\forall \bar{x}_{1}, \bar{x}_{2}\right) D\left(\bar{x}_{1}, \bar{x}_{2}\right)$ from (66) relates to the degree of inclusion, see (44).

Now, we will investigate a relationship between the precise value of $F$ and conclusion $B^{*}$ of the inferences based on the approximate description.

Theorem 9 Let $P_{1} \equiv_{d f} \operatorname{Ext}_{\bar{R}} F \& C_{k}$ and $P_{2} \equiv_{d f}\left(\operatorname{Ext}_{\bar{R}} F\right)^{3} \& C_{k} \& A^{*}\left(\bar{x}_{p}\right)$. Then

$$
\begin{aligned}
\operatorname{Ext}_{\bar{R}} F & \rightarrow\left[\left(\forall \bar{x}_{p}\right) A^{*}\left(\bar{x}_{p}\right) \&\left(\forall \bar{y}_{p}\right) B_{\mathrm{DNF}}^{*}\left(\bar{y}_{p}\right) \rightarrow\left(\exists \bar{x}_{p}\right)\left(\forall \bar{y}_{p}\right) F(\bar{x})\right], \\
\operatorname{Ext}_{\bar{R}} F & \rightarrow\left[(\forall \bar{x}) F(\bar{x}) \rightarrow\left(\left(\forall \bar{x}_{p}\right) A^{*}\left(\bar{x}_{p}\right) \rightarrow\left(\forall \bar{y}_{p}\right) B_{\mathrm{CNF}}^{*}\left(\bar{y}_{p}\right)\right)\right], \\
P_{1} & \rightarrow F(\bar{x}) \subseteq\left(A^{*}\left(\bar{x}_{p}\right) \rightarrow B_{\mathrm{DNF}}^{*}\left(\bar{y}_{p}\right)\right), \\
P_{1} & \rightarrow\left(A^{*}\left(\bar{x}_{p}\right) \& B_{\mathrm{CNF}}^{*}\left(\bar{y}_{p}\right)\right) \subseteq F(\bar{x}), \\
P_{2} & \rightarrow\left[F(\bar{x}) \leftrightarrow B_{\mathrm{DNF}}^{*}\left(\bar{y}_{p}\right)\right], \\
P_{2} & \rightarrow\left[F(\bar{x}) \leftrightarrow B_{\mathrm{CNF}}^{*}\left(\bar{y}_{p}\right)\right] .
\end{aligned}
$$

\section{PROOF:}


(70) Starting from (53) and using (4), we derive

$$
\operatorname{Ext}_{\bar{R}} F \rightarrow\left[\left(\forall \bar{x}_{p}\right) A^{*}\left(\bar{x}_{p}\right) \& B_{\mathrm{DNF}}^{*}\left(\bar{y}_{p}\right) \rightarrow\left(\exists \bar{x}_{p}\right) F(\bar{x})\right]
$$

It remains to generalize and distribute the universal quantifiers.

(71) Analogously, we take (54), apply (4) and adjunction (6), which gives

$$
\operatorname{Ext}_{\bar{R}} F \rightarrow\left[\left(\forall \bar{x}_{p}\right) F(\bar{x}) \rightarrow\left(\left(\forall \bar{x}_{p}\right) A^{*}\left(\bar{x}_{p}\right) \rightarrow B_{\mathrm{CNF}}^{*}\left(\bar{y}_{p}\right)\right)\right]
$$

The generalization and distribution of the quantifiers will give the result formula.

(72) Let $C_{k}(\bar{x})$ denote $\bigvee_{i \in I_{k}}\left(R\left(\bar{x}, \bar{c}_{i}\right) \& R\left(\bar{c}_{i}, \bar{x}\right)\right)$. From $(55)$

$$
\left(\operatorname{Ext}_{\bar{R}} F \& C_{k}(\bar{x})\right) \& F(\bar{x}) \& A^{*}\left(\bar{x}_{p}\right) \rightarrow\left(\exists \bar{x}_{p}\right)\left(A^{*}\left(\bar{x}_{p}\right) \& \operatorname{DNF}(\bar{x})\right)
$$

and using adjunction.

(73) Analogously.

(74) - (75) Obviously by (68).

Since $F$ represents some real situation that is to be approximately described by approximating formulae, we wish to know the relationship w.r.t. $B_{\mathrm{D}(\mathrm{C}) \mathrm{NF}}^{*}$ that has been investigated in the above lemma. From $P_{1,2}$ it follows that besides extensionality we need an appropriate partition, i.e. the distribution of input and output fuzzy sets associated to $\bar{R}(\overline{\mathbf{c}}, \bar{x}), \bar{R}(\bar{x}, \overline{\mathbf{c}})$, that lead to the indistinguishability between $B_{\mathrm{D}(\mathrm{C}) \mathrm{NF}}^{*}$ and $F$.

Theorem 10 Let us denote $\left[c_{i_{p+1}}, \ldots, c_{i_{n}}\right]$ by $\bar{d}_{i_{p}}$ and let

$$
\begin{aligned}
\operatorname{Disj}(R) & \equiv_{d f}\left(\forall \bar{x}_{p}\right) \bigwedge_{i \neq j} \neg\left[R\left(\bar{x}_{p}, \bar{c}_{i_{p}}\right) \& R\left(\bar{x}_{p}, \bar{c}_{j_{p}}\right)\right], \\
L_{1} & \equiv_{d f}\left[R\left(\bar{c}_{i_{p}}, \bar{x}_{p}\right) \subseteq A^{*}\left(\bar{x}_{p}\right)\right] \&\left(\exists \bar{x}_{p}\right) R^{2}\left(\bar{c}_{i_{p}}, \bar{x}_{p}\right), \\
L_{2} & \equiv_{d f}\left[R\left(\bar{x}_{p}, \bar{c}_{i_{p}}\right) \subseteq A^{*}\left(\bar{x}_{p}\right)\right] \&\left(\exists \bar{x}_{p}\right) R^{2}\left(\bar{x}_{p}, \bar{c}_{i_{p}}\right), \\
L_{3} & \equiv_{d f}\left[A^{*}\left(\bar{x}_{p}\right) \subseteq R\left(\bar{c}_{i_{p}}, \bar{x}_{p}\right)\right] \& \operatorname{Disj}(R), \\
L_{4} & \equiv_{d f}\left[A^{*}\left(\bar{x}_{p}\right) \subseteq R\left(\bar{x}_{p}, \bar{c}_{i_{p}}\right)\right] \& \operatorname{Disj}(R), \\
L_{5} & \equiv_{d f}\left[A^{*}\left(\bar{x}_{p}\right) \approx R\left(\bar{c}_{i_{p}}, \bar{x}_{p}\right)\right] \&\left(\left(\exists \bar{x}_{p}\right) R^{2}\left(\bar{c}_{i_{p}}, \bar{x}_{p}\right) \wedge \operatorname{Disj}(R)\right), \\
L_{6} & \equiv_{d f}\left[A^{*}\left(\bar{x}_{p}\right) \approx R\left(\bar{x}_{p}, \bar{c}_{i_{p}}\right)\right] \&\left(\left(\exists \bar{x}_{p}\right) R^{2}\left(\bar{x}_{p}, \bar{c}_{i_{p}}\right) \wedge \operatorname{Disj}(R)\right) .
\end{aligned}
$$


Then

$$
\begin{aligned}
& L_{1} \rightarrow\left[R\left(\bar{d}_{i_{p}}, \bar{y}_{p}\right) \& F\left(\bar{c}_{i}\right)\right] \subseteq B_{\mathrm{DNF}}^{*}\left(\bar{y}_{p}\right), \\
& L_{2} \rightarrow B_{\mathrm{CNF}}^{*}\left(\bar{y}_{p}\right) \subseteq\left[R\left(\bar{y}_{p}, \bar{d}_{i_{p}}\right) \rightarrow F\left(\bar{c}_{i}\right)\right], \\
& L_{3} \rightarrow B_{\mathrm{DNF}}^{*}\left(\bar{y}_{p}\right) \subseteq\left[R\left(\bar{d}_{i_{p}}, \bar{y}_{p}\right) \& F\left(\bar{c}_{i}\right)\right], \\
& L_{4} \rightarrow\left[R\left(\bar{y}_{p}, \bar{d}_{i_{p}}\right) \rightarrow F\left(\bar{c}_{i}\right)\right] \subseteq B_{\mathrm{CNF}}^{*}\left(\bar{y}_{p}\right), \\
& L_{5} \rightarrow B_{\mathrm{DNF}}^{*}\left(\bar{y}_{p}\right) \approx\left[R\left(\bar{d}_{i_{p}}, \bar{y}_{p}\right) \& F\left(\bar{c}_{i}\right)\right], \\
& L_{6} \rightarrow B_{\mathrm{CNF}}^{*}\left(\bar{y}_{p}\right) \approx\left[R\left(\bar{y}_{p}, \bar{d}_{i_{p}}\right) \rightarrow F\left(\bar{c}_{i}\right)\right] .
\end{aligned}
$$

\section{PROOF:}

(76) Let us denote $R\left(\bar{d}_{i_{p}}, \bar{y}_{p}\right) \& F\left(\bar{c}_{i}\right)$ by $B\left(\bar{y}_{p}\right)$

$$
\begin{aligned}
& \left(\exists \bar{x}_{p}\right) R^{2}\left(\bar{c}_{i_{p}}, \bar{x}_{p}\right) \&\left(\forall \bar{x}_{p}\right)\left(R\left(\bar{c}_{i_{p}}, \bar{x}_{p}\right) \rightarrow A^{*}\left(\bar{x}_{p}\right)\right) \& B\left(\bar{y}_{p}\right) \longrightarrow \\
& \quad\left(\exists \bar{x}_{p}\right)\left[R^{2}\left(\bar{c}_{i_{p}}, \bar{x}_{p}\right) \&\left(R\left(\bar{c}_{i_{p}}, \bar{x}_{p}\right) \rightarrow A^{*}\left(\bar{x}_{p}\right)\right) \& B\left(\bar{y}_{p}\right)\right] \longrightarrow \text { by }(15) \\
& \left(\exists \bar{x}_{p}\right)\left[A^{*}\left(\bar{x}_{p}\right) \& R\left(\bar{c}_{i}, \bar{x}\right) \& F\left(\bar{c}_{i}\right)\right] \longrightarrow \\
& \quad\left(\exists \bar{x}_{p}\right)\left[A^{*}\left(\bar{x}_{p}\right) \& \bigvee_{j \in I}\left(R\left(\bar{c}_{j}, \bar{x}\right) \& F\left(\bar{c}_{j}\right)\right)\right] \longrightarrow B_{\mathrm{DNF}}^{*}\left(\bar{y}_{p}\right) .
\end{aligned}
$$

(77) Analogous to (76).

(78)

$$
\begin{aligned}
\varphi \longleftrightarrow & B_{\mathrm{DNF}}^{*}\left(\bar{y}_{p}\right) \&\left[A^{*}\left(\bar{x}_{p}\right) \subseteq R\left(\bar{c}_{i_{p}}, \bar{x}_{p}\right)\right] \longrightarrow \\
& \left(\exists \bar{x}_{p}\right)\left[A^{*}\left(\bar{x}_{p}\right) \& \bigvee_{j \in I}\left(R\left(\bar{c}_{j}, \bar{x}\right) \& F\left(\bar{c}_{j}\right)\right)\right] \&\left[A^{*}\left(\bar{x}_{p}\right) \rightarrow R\left(\bar{c}_{i_{p}}, \bar{x}_{p}\right)\right] \longrightarrow \\
& \left(\exists \bar{x}_{p}\right) R\left(\bar{c}_{i_{p}}, \bar{x}_{p}\right) \& \bigvee_{j \in I}\left(R\left(\bar{c}_{j}, \bar{x}\right) \& F\left(\bar{c}_{j}\right)\right) \longleftrightarrow \psi
\end{aligned}
$$

Since $\operatorname{Disj}(R) \leftrightarrow\left(\left(\exists \bar{x}_{p}\right) \bigvee_{i \neq j}\left[R\left(\bar{x}_{p}, \bar{c}_{i_{p}}\right) \& R\left(\bar{x}_{p}, \bar{c}_{j_{p}}\right)\right] \rightarrow \overline{0}\right)$, it follows that

$$
\begin{aligned}
& \operatorname{Disj}(R) \& \varphi \longrightarrow \psi \longrightarrow \\
& \left(\exists \bar{x}_{p}\right) R^{2}\left(\bar{c}_{i_{p}}, \bar{x}_{p}\right) \& R\left(\bar{d}_{i_{p}}, \bar{y}_{p}\right) \& F\left(\bar{c}_{i}\right) \longrightarrow R\left(\bar{d}_{i_{p}}, \bar{y}_{p}\right) \& F\left(\bar{c}_{i}\right),
\end{aligned}
$$

which gives the result by moving $B_{\mathrm{DNF}}^{*}\left(\bar{y}_{p}\right)$ on the right side, generalizing by $\bar{y}_{p}$ and moving the quantifier due to (11).

(79) Analogous to (78).

$(80)-(81)$ Since $(\varphi \approx \psi) \&(\alpha \wedge \beta) \longleftrightarrow[(\varphi \approx \psi) \& \alpha] \wedge[(\varphi \approx \psi) \& \beta] \longrightarrow[(\varphi \subseteq$ $\psi) \& \alpha] \wedge[(\varphi \subseteq \psi) \& \beta]$ then obviously by $L_{5(6)} \rightarrow L_{1(2)} \wedge L_{3(4)} \longrightarrow$ the right 


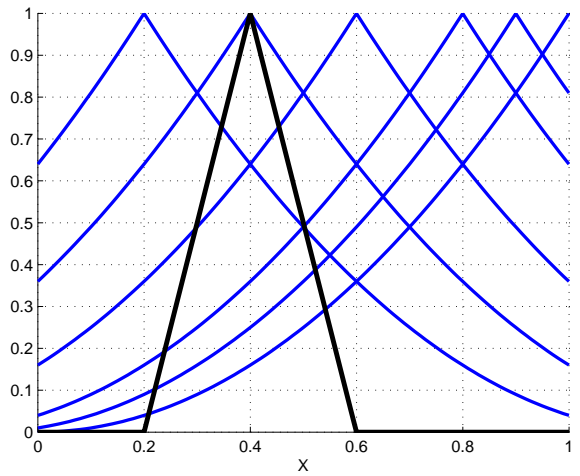

(a) Input fuzzy number (black line) assigned to $A^{*}$ and fuzzy sets assigned to $R_{1}\left(c_{i_{1}}, x\right), i \in I$

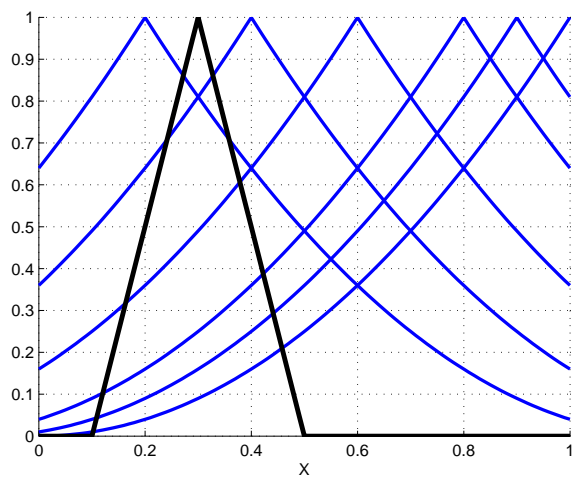

(c) Analogous to (a) with the shifted fuzzy number (black line)

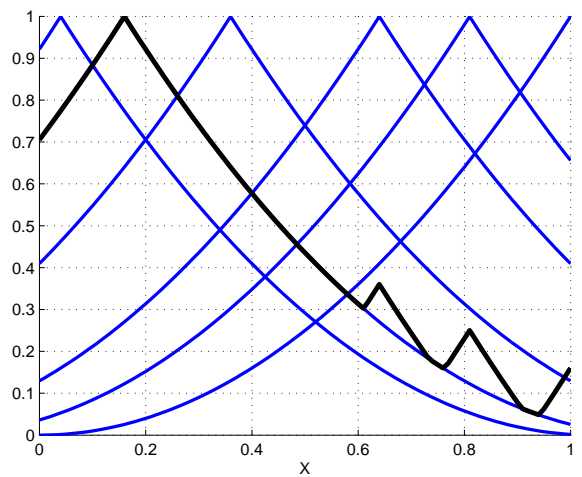

(b) Output fuzzy set determined by $B_{\mathrm{DNF}}^{*}$ (black line) based on $A^{*}$ from (a) and fuzzy sets assigned to $R_{2}\left(\mathbf{c}_{i_{2}}, x\right), i \in I$

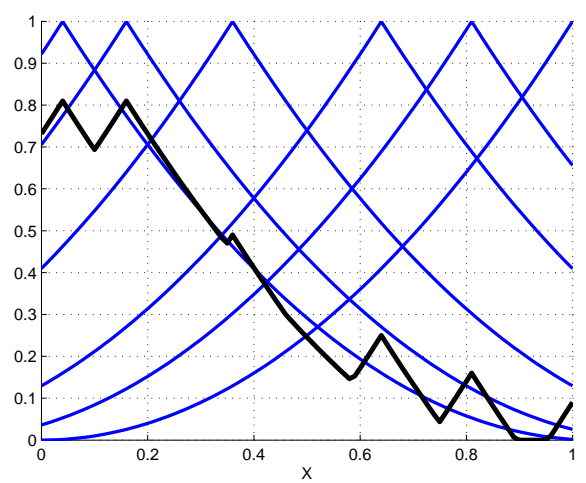

(d) Analogous to (b), where $A^{*}$ is due to (c)

Fig. 7. Inference with DNF and triangular fuzzy number.

side of the respective implication.

In order to understand the proved relationships (76)-(81), it is worth to pay attention to the following formulae:

$$
\begin{array}{cl}
\left(\exists \bar{x}_{p}\right) R^{2}\left(\bar{c}_{i_{p}}, \bar{x}_{p}\right), & \left(\exists \bar{x}_{p}\right) R^{2}\left(\bar{x}_{p}, \bar{c}_{i_{p}}\right), \\
\bigwedge_{i \neq j} \neg\left[R\left(\bar{c}_{i_{p}}, \bar{x}_{p}\right) \& R\left(\bar{c}_{j_{p}}, \bar{x}_{p}\right)\right], & \bigwedge_{i \neq j} \neg\left[R\left(\bar{x}_{p}, \bar{c}_{i_{p}}\right) \& R\left(\bar{x}_{p}, \bar{c}_{j_{p}}\right)\right] .
\end{array}
$$

Let $\left\langle\left\{\tilde{R}_{j}\right\}_{j \in J},\left\{c_{i}\right\}_{i \in I}\right\rangle$ be a model for $\left\langle\left\{R_{j}\right\}_{j \in J},\left\{\bar{c}_{i}\right\}_{i \in I}\right\rangle$, where $j \in\{1, \ldots, n\}$. Then each formula in (82) determines the height of a fuzzy set describing "very close" neighborhood of $c_{i}$ being a subset of $\tilde{R}\left(c_{i}, x\right)$ or $\tilde{R}\left(x, c_{i}\right)$ that represents this neighborhood. And formula (83) says how much $\tilde{R}_{i}\left(c_{i}, x\right)$ is disjoint from all the others $\tilde{R}_{j}\left(c_{j}, x\right)$ (analogously for $\tilde{R}_{i}\left(x, c_{i}\right)$ ).

Example 7 We will continue with Example 6.7 and demonstrate how inferences with $\mathrm{DNF}$ and $\mathrm{CNF}$ work in this setting. 


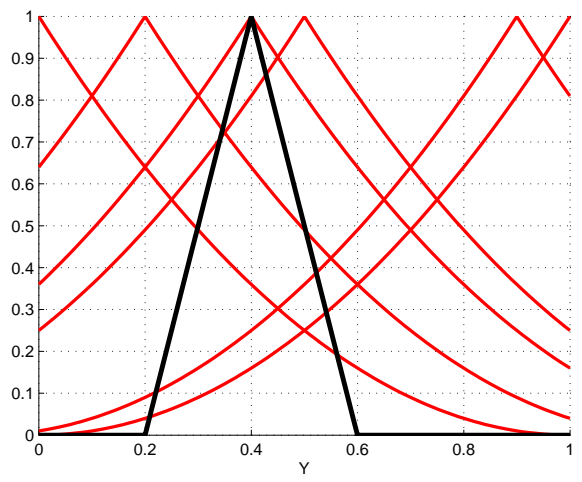

(a) Input fuzzy number (black line) assigned to $A^{*}$ and fuzzy sets assigned to $R_{1}\left(x, c_{i_{1}}\right), i \in I$

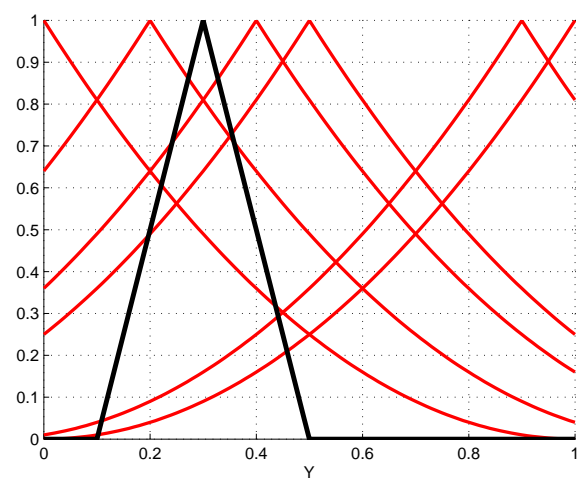

(c) Analogous to (a) with the shifted fuzzy number (black line)

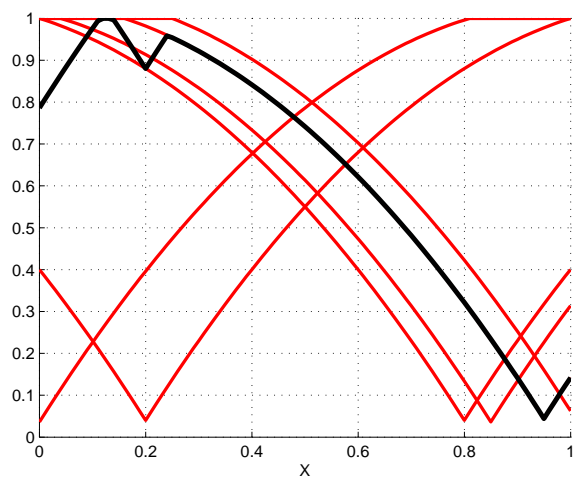

(b) Output fuzzy set determined by $B_{\mathrm{CNF}}^{*}$ (black line) based on $A^{*}$ from (a) and fuzzy sets assigned to $R_{2}\left(x, \mathbf{c}_{i_{2}}\right), i \in I$

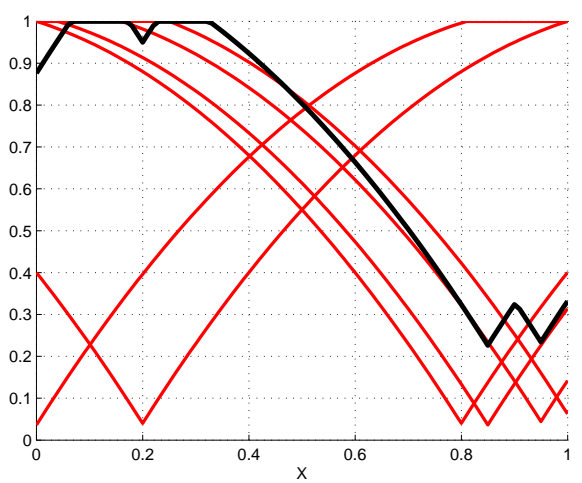

(d) Analogous to (b), where $A^{*}$ is due to (c)

Fig. 8. Inference with CNF and triangular fuzzy number.

Figures 7 and 8 illustrates formulae from Theorem 10. From (a) + (b), we see that whenever $A^{*}$ is contained in one of the left sides of the rules then the output nearly coincides with the right side of this particular rule. Subfigures (c) $+(d)$ demonstrate that if $A^{*}$ is not subset of any of the left-hand sides of the rules then also the output fuzzy set reflects this. We may take it as a lack of knowledge included in $\mathrm{D}(\mathrm{C}) \mathrm{NF}$ that is transferred to $B^{*}$.

\section{Conclusions}

The results of this paper shed light on the problems of approximate reasoning based on an imprecise description of some real physical system. Therefore, this work contributes to the theory of fuzzy systems. Especially the formulae from Section 3.2 provide a quality estimation of fuzzy control system consisting of rule-base interpreted as DNF or CNF and appropriate inference rule. The 
traditional approach with Mamdani's type of the rules and Zadeh's compositional rule of inference has been extended by graded rules of the type $\mathrm{D}(\mathrm{C}) \mathrm{NF}$ together with the compositional rule of inference $B_{\mathrm{D}(\mathrm{C}) \mathrm{NF}}^{*}$. The dual systems (deductive and abductive) were build in parallel.

Acknowledgement The author is grateful to Professor Vilém Novák for his pertinent comments that significantly improved this article. Thanks go also to Libor Běhounek for his helpful suggestions.

This investigation has been supported by project MSM6198898701 of the MŠMT ČR.

\section{References}

[1] K.-E. Årzén, M. Johansson, R. Babuška, Fuzzy control versus conventional control, in: H. Verbruggen, H.-J. Zimmermann, R. Babuška (Eds.), Fuzzy Algorithms for Control, Boston, Kluwer, 1999, pp. 111-125.

[2] W. Bandler, L. Kohout, Special properties, closures and interiors of crisp and fuzzy relations, Fuzzy Sets and Systems 26 (1988) 317-331.

[3] L. Běhounek, P. Cintula, From fuzzy logic to fuzzy mathematics: A methodological manifesto, Fuzzy Sets and Systems 157 (2006) 642-646.

[4] R. Bělohlávek, Fuzzy Relational Systems: Foundations and Principles, Kluwer Academic/Plenum Press (Vol. 20 of IFSR Int. Series on Systems Science and Engineering), New York, 2002.

[5] U. Bodenhofer, A unified framework of opening and closure operators with respect to arbitrary fuzzy relations, Soft Computing 7 (2003) 220-227.

[6] D. Boixader, J. Jacas, Extensionality based approximate reasoning, Int. Journal of Approximate Reasoning 19 (1998) 221-230.

[7] D. Butnariu, E.P. Klement, S. Zafrany, On triangular norm based propositional fuzzy logics, Fuzzy Sets and Systems 69 (1995) 241-255.

[8] P. Cintula, Basics of A Formal Theory of Fuzzy Partitions, in: Proceedings of EUSFLAT-LFA 2005, 2005, pp. 884-888.

[9] P. Cintula, The $€ \Pi$ and $€ \Pi \frac{1}{2}$ propositional and predicate logics, Fuzzy Sets and Systems 124 (2001) 289-302.

[10] M. Daňková, I. Perfilieva, Logical Approximation II, Soft Computing 7 (2003) $228-233$.

[11] D. Dubois, J. Lang, H. Prade, Fuzzy sets in approximate reasoning, Part 2: logical approaches, Fuzzy Sets and Systems 40 (1991) 203-244. 
[12] D. Dubois , H. Prade, Fuzzy sets in approximate reasoning, part 1: inference with possibility distributions, Fuzzy Sets and Systems 40 (1991) 143-202.

[13] D. Dubois, H. Prade, F. Esteva, P. Garcia, L. Godo, A logical approach to interpolation based on similarity relations, International Journal of Approximate Reasoning 17 (1997) 1-36.

[14] D. Dubois, H. Prade, L. Ughetto, Fuzzy Logic, Control Engineering and Artificial Intelligence, in: H. Verbruggen, H.-J. Zimmermann, R. Babuška (Eds.), Fuzzy Algorithms for Control, Boston, Kluwer, 1999, pp. 17-58.

[15] S. Gottwald, A Treatise on Many-Valued Logics. Studies in Logic and Computation, Vol. 9, Research Studies Press: Baldock, Hertfordshire, England, 2001.

[16] S. Gottwald, Fuzzy Sets and Fuzzy Logic: Foundations of Applicationfrom a Mathematical Point of View, Vieweg, Wiesbaden, 1993.

[17] P. Hájek, Metamathematics of fuzzy logic, Kluwer, Dordrecht, 1998.

[18] F. Klawonn, V. Novák, The relation between inference and interpolation in the framework of fuzzy systems, Fuzzy sets and systems 81 (1996) 331-354.

[19] V. Novák, S. Lehmke, Logical structure of fuzzy IF-THEN rules, Fuzzy Sets and Systems 157 (2006) 2003-2029.

[20] V. Novák, On the logical basis of approximate reasoning, in: V. Novák, J. Ramík, M. Mareš, M. Černý, J. Nekola (Eds.), Fuzzy Approach to Reasoning and Decision Making, Kluwer, Dordrecht, 1992.

[21] V. Novák, I. Perfilieva, J. Močkoř, Mathematical Principles of Fuzzy Logic, Kluwer, Boston Dordrecht, 1999.

[22] I. Perfilieva, Logical Approximation, Soft Computing 2 (2001) 73-78.

[23] I. Perfilieva, Logical Foundations of Rule-Based Systems, Fuzzy Sets and Systems 157 (2006) 615-621.

[24] E. Tsiporkova-Hristoskova, B. De Baets, E. Kerre, A detailed study of direct and inverse images under fuzzy multivalued mappings, The Journal of Fuzzy Mathematics 3 (1995) 191-208.

[25] G.-J. Wang, Formalized theory of general fuzzy reasoning, Information SciencesInformatics and Computer Science 160 (2004) $251-266$.

[26] L.A. Zadeh, A theory of approximate reasoning, in: J.E. Hayes, D. Mitchie, L.I. Mikulich (Eds.), Machine Intelligence (9), Wiley, New York, 1979, pp. 149-194.

[27] L.A. Zadeh, Outline of a new approach to the analysis of complex systems and decision processes, IEEE Transactions on Systems, Man and Cybernetics 3 (1973) 28-44. 